\title{
Holistic Characterization of Tumor Monocyte-to-Macrophage Differentiation Integrates Distinct Immune Phenotypes in Kidney Cancer
}

Adriana M. Mujal ${ }^{1,6,7}$, Alexis J. Combes ${ }^{1,2,3,7}$, Arjun R. Rao ${ }^{2,3,8}$, Mikhail Binnewies ${ }^{1,8}$, Bushra Samad $^{1,2,3}$, Jessica Tsui ${ }^{1,2,3}$, Alexandre Boissonnas $^{4}$, Joshua L. Pollack ${ }^{3}$, Rafael J. Argüello $^{5}$, Megan K. Ruhland ${ }^{1}$, Kevin C. Barry ${ }^{1}$, Vincent Chan ${ }^{1,2,3}$

\& Matthew F. Krummel ${ }^{1,2,3,9 \ddagger}$

${ }^{1}$ Department of Pathology and ImmunoX, ${ }^{2}$ UCSF CoLabs, ${ }^{3}$ UCSF Immunoprofiler Initiative, San Francisco, CA 94143, USA

${ }^{4}$ Sorbonne Université, INSERM, CNRS, Centre d'Immunologie et des Maladies Infectieuses Cimi-Paris, F-75013, Paris, France

${ }^{5}$ Aix Marseille Univ, CNRS, INSERM, CIML, Centre d'Immunologie de Marseille-Luminy, Marseille, France

${ }^{6}$ Present address: Immunology Program, Memorial Sloan Kettering Cancer Center, New York, NY 10065, USA

${ }^{7}$ These authors contributed equally to this work

${ }^{8}$ These authors contributed equally to this work

${ }^{9}$ Lead contact

‡Correspondence: matthew.krummel@ucsf.edu 


\section{Abstract}

The tumor immune microenvironment (TIME) is commonly infiltrated by diverse collections of myeloid cells. Yet, the complexity of myeloid cell identity and plasticity has challenged efforts to define bona fide populations and determine their connections to $T$ cell function and their relation to patient outcome. Here we leverage single-cell RNA-sequencing (scRNA-seq) analysis of several mouse and human tumors and find that monocyte-macrophage diversity is characterized by a combination of conserved lineage states as well as transcriptional programs accessed along the differentiation trajectory. Using mouse models, we also find that tumor monocyte-to-macrophage progression is profoundly tied to regulatory $T$ cell $\left(T_{\text {reg }}\right)$ abundance. Importantly, in human kidney cancer, heterogeneity in macrophage accumulation and myeloid composition corresponded to variance in, not only $T_{\text {reg }}$ density, but also the quality of infiltrating $\mathrm{CD}^{+} \mathrm{T}$ cells. In this way, holistic analysis of monocyte-to-macrophage differentiation creates a framework for critically different immune states in kidney tumors. 


\section{Introduction}

A key component of most immune responses, including those to cancers, are mononuclear phagocyte cell populations, which share common features of phagocytosis, tissue repair, and immunoregulation but diverge in functional specialization. Conventional dendritic cells (cDCs) are positioned in tissues to initiate and sustain adaptive $T$ cell responses ${ }^{1,2}$ while macrophages (MFs) engage in high rates of phagocytosis and tissue remodeling ${ }^{3-5}$. Self-renewing tissueresident macrophages are seeded during embryonic development ${ }^{6-10}$, while inflammatory stimuli prompt infiltration of adult hematopoietic stem cell-derived monocytes that give rise to tumor macrophages ${ }^{11-14}$. These monocyte-derived macrophages preferentially accumulate as tumors progress $^{15}$ and may predominate in regulating the ongoing antitumor T cell response ${ }^{16}$.

Macrophages consist of numerous subset populations that have been identified across tissues ${ }^{17-20}$. Therapeutic blockade of key epigenetic and signaling pathways has demonstrated their amenability to transcriptional reprogramming ${ }^{21-27}$, but how phenotypic diversity arises still remains poorly understood. Recruited bloodborne monocytes exhibit plasticity in differentiation potential and can acquire features of macrophages and/or DCs depending on the inflammatory setting $^{12,13,17,28-32}$. In addition, early studies demonstrated that macrophage exposure to type 1or type 2-associated cytokines induces "M1" or "M2" cellular programs, respectively, and a model was put forth in which myeloid cells are polarized to be pro- (“M1") or anti- ("M2") inflammatory ${ }^{33-35}$. Although this nomenclature was thereafter understood to require nuance to account for additional plasticity ${ }^{36}$, it remains undetermined if these binary programs are applicable to describe tumor macrophage differentiation in vivo.

Myeloid phenotypic diversity has also challenged efforts to utilize myeloid populations as biomarkers for patient treatment options and outcome. cDCs are critical for coordinating antitumor T cell immunity ${ }^{37-42}$ and higher CDC abundance is broadly associated with improved 
cancer patient survival, although additional TIME features may inform functionality ${ }^{38,39,43}$. In contrast, macrophages have largely been considered to be pro-tumoral ${ }^{5,44}$ and monocytes have often been described as myeloid-derived suppressor cells (MDSCs) ${ }^{45}$. Yet, several studies have exhibited variability in the use of macrophages as a negative predictor of patient prognosis ${ }^{46-49}$, and increased levels of circulating monocytes were unexpectedly linked to patient responsiveness to immune checkpoint blockade $(\mathrm{ICB})^{50}$. These contrary findings speak to the need for improved resolution of myeloid cell categorization and phenotype in order to dissect heterogenous responses amongst cancer patients.

We used scRNA-seq to uncover transcriptional heterogeneity amongst tumor-infiltrating myeloid cells and distinguished monocyte and macrophage lineage- and activation-induced programs shared between multiple mouse tumor models and human kidney cancer samples. Monocyte differentiation is dynamically regulated, and we found that $T_{\text {reg }}$ density is one example of an immunoregulatory axis that can modulate macrophage density. Further comprehensive analysis of key myeloid populations revealed distinct network connections between different myeloid cell types and $T$ cell subsets, including $T_{\text {regs }}$ and effector $T$ cells. This is consistent with an archetypal organization of immune systems in tumors - collections of cell types that move together as modules ${ }^{51}$ - and improved classification of patients such that we could identify those with effective antitumor $\mathrm{T}$ cell responses. 


\section{Results}

\section{Establishing Diversity of Differentiation and Environment-responsive Myeloid States in Mouse B16 Tumors}

Subcutaneous implantation of B16 melanoma cells is a well-established mouse tumor model with abundant infiltration of monocytes, macrophages, and $\mathrm{CDCs}^{38}$. To study these cells along their differentiation trajectories, we used conventional markers to sort bulk myeloid populations, along with reference sorted populations of $\mathrm{Ly}_{6 \mathrm{C}} \mathrm{C}^{+}$monocytes and two tumor-associated (TAM) populations, distinguished based on expression level of CD11C and MHC-II ${ }^{38}$ (Fig 1A, S1A), and then subjected all of these to scRNA-seq analysis.

Within the bulk myeloid population, $t$-SNE clustering yielded eight transcriptionallydistinct cell populations (Fig. 1B, S1B-C), including three Flt3 ${ }^{+} \mathrm{Kit}^{+} \mathrm{cDC}$ populations (Clusters 4, $6,7)$, which were marked by signatures specific to $c D C 1 s, c D C 2 s$, and conserved $c D C$ activation programs (Fig. S1D, Table S1) ${ }^{38,39,52}$. The remaining myeloid cells (Clusters $0,1,2,3$, 5) broadly expressed Csf1r and Mafb (Fig. 1C), indicative of monocytes and macrophages. Having focused on the stimulatory capacity of $\mathrm{CDC}$ in previous work ${ }^{38,39,53}$, here we focused on the diversity of monocytes and macrophages as it related to the TIME.

To align transcriptional cell type categorization with flow cytometry analysis, we generated cell-type-specific gene signatures from the scRNA-seq analysis of the FACS-sorted monocytes and TAMs (Fig. 1A, S1E). When applied (Fig. 1D), these indicated that four Csf1r ${ }^{+}$ $\mathrm{Mafb}^{+}$populations (Clusters $\left.0,1,2,5\right)$ expressed monocyte-specific genes, an unexpected heterogeneity. The four monocyte populations expressed $L y 6 c 2$, but varied in levels of other monocyte-associated genes (e.g., $\mathrm{Hp}, \mathrm{Chil3}$ ) and, as found in Cluster 0, also expressed TAM- 
associated genes (e.g. H2-Ab1, C1qa, Ms4a7) (Fig. 1E-F, S1E). Monocyte-like clusters were differentiated from one another by cellular activation programs. For example, Cluster 1 ("IFNresponsive") was specifically enriched for interferon (IFN)-inducible genes such as Cxc/10, Gbp2, and IFIT-family members. Cluster 2 ("stress-responsive") cells expressed Arg1 and were enriched for cellular stress processes, including oxidative stress-responsive genes and heatshock protein genes such as Hmox1, Hspa1a, Hilpda, Bnip3, Ero1l, and Ndrg1 (Fig. 1F, S1F). In contrast to the heterogeneity observed amongst monocytes, signatures for both populations of TAMs localized within Cluster 3 (Fig. 1D).

We applied pseudotime analysis ${ }^{54}$ to generate a model of tumor monocyte-tomacrophage differentiation (Fig. 1G-H, S1G). This model placed Cluster $5 \mathrm{Ly} 6 \mathrm{c} 2^{+} H \mathrm{p}^{+}$ monocytes and Cluster $3 \mathrm{C}_{\mathrm{qqa}}{ }^{+}$TAMs at opposite ends of a linear trajectory consistent with expectations. Cluster 0 monocytes occupied the continuum between them and expressed a combination of both monocyte- and TAM-associated signatures such that we designated these cells "Intermediate monocytes" (“Mono-Int”). Kinetic analysis of cluster-enriched genes confirmed gradual downregulation of $\mathrm{Ly}_{6} \mathrm{c} 2^{+} \mathrm{Hp}^{+}$monocyte-associated genes and up-regulation of "Mono-Int"- and TAM-associated genes along the pseudotime trajectory (Fig. 1I). This transcriptional model thus supported a framework of progressive monocyte-to-TAM differentiation, in which Ly6C down-regulation is paired with up-regulation of CD64, MHC-II, and F4/80 28 (Fig. S1H-I). In contrast, IFN- and stress-responsive cells occupied distinct branches that diverged from the dominant differentiation trajectory at intermediate timepoints (Fig. 1H-I, S1G). 


\section{ScRNA-seq Highlights Heterogeneous Acquisition of 'Stress-' and 'Interferon- Responsive' Cellular Programs during Tumor Monocyte-to-Macrophage Differentiation}

To gain higher resolution on the differentiation trajectories within this lineage, we next performed cluster analysis on the sorted monocyte and TAM samples. Sorted monocytes expressed $L y 6 c 2$ and contained clusters similar to those identified within the bulk myeloid cell sample (Fig. 2A, S2A). Cluster analysis of CD11 $\mathrm{C}^{\text {lo }}$ and CD11 $\mathrm{C}^{\mathrm{hi}}$ TAMs, however, resolved diversity beyond the C1qa ${ }^{+}$TAM signature (Fig. 2B, S2B-C) including clusters enriched for cell cycle-related genes, and an $M g / 2^{+}$TAM subset that expressed immune modulators such as $C c / 6, I / 1 b$, and Retnla as compared to the C1qa ${ }^{+}$cluster which more highly expressed genes such as Ms4a7. Although these cells had not formed a distinct population in our original analysis of bulk myeloid cells (Fig. 1), we did retrospectively detect $M g / 2^{+}$cells in in that scRNA-seq data, as well as by flow cytometry (Fig. S2D). TAM-subset clusters were surprisingly also accompanied by an $\mathrm{Arg} 1^{+}$ stress-responsive cluster akin to that found in the sorted monocytes (Fig. 2B, S2B-C). Indeed, re-clustering of the entire stress-responsive cluster from the bulk tumor myeloid sample revealed that this program was acquired by monocytes, "Mono-Int" and TAMs (Fig. 2C, S2E).

Segregated expression of stress-responsive genes and canonical TAM-associated genes suggested divergent transcriptional programs and we sought to determine if these populations could also be distinguished by flow cytometry. Differential gene expression analysis of the stress-responsive and C1qa+ TAM clusters from our bulk myeloid cell sample revealed cluster-specific expression of cell surface genes $I / 7 r$ and Vcam1, respectively (Fig. 2D). Using the same gating as in Fig. S1A, we confirmed this split in both "Mono-Int" and TAMs (Fig. 2E) and we found enriched arginase 1 (ARG1) expression in both IL-7R $\alpha^{+}$populations (Fig. 2E-F, S2F). As expected from the single-cell transcriptional analysis, VCAM1 ${ }^{+}$cells were more abundantly found within TAMs (Fig. 2E-F, S2F). 
Together, this dissection of sorted cell populations lent support to a model in which monocytes and TAMs exist in a differentiation trajectory, along which cells can adopt specialized cellular programs (Fig. 2G-H). Some programs, such as those associated with $M g / 2^{+}$or Vcam $^{+}$TAMs, selectively emerged later, in mature TAMs. Others, such as IFNinduced signaling or stress-responsiveness may be more universally accessible across differentiation stages. Interestingly, we detected populations of IFN-responsive monocytes in the peripheral blood of B16 tumor-bearing mice (Fig. S2G-H), perhaps suggesting that systemic IFN signaling, or other induction of this program, may define monocytes prior to tumor entry. In contrast, stress-responsive populations were not detected in the blood, suggesting that microenvironmental cues in the TIME likely induce this activation program locally. Further studies are warranted to explore if these programs directly influence monocyte differentiation processes or act as 'layers' that accessorize a canonical differentiation trajectory.

\section{Mouse Tumor Macrophage Subset Heterogeneity Does Not Reflect “M1/M2” Polarization}

Macrophage exposure to type-1 or type-2 cytokines in vitro results in "M1" and "M2" transcriptional signatures that are often used to describe 'pro-inflammatory,' or 'antiinflammatory' and wound healing processes, respectively ${ }^{33-35}$. To address whether "M1/M2" polarization was a useful construct to define tumor macrophage diversity in vivo, we tested how "M1" and "M2" gene signatures ${ }^{55}$ corresponded to tumor myeloid cell subsets profiled here. Using correlation and clustering analyses (Fig. 3A, S3A), we found that, contrary to in vitro findings, tumor myeloid cells were marked by broad expression of both "M1"- and "M2"associated genes, and we did not observe substantial correlation of gene expression within "M1" or "M2" gene groups across single cells. These data suggest that while tumor myeloid cells 
can express individual "M1" and "M2" genes, they rarely do so in any distinguishably consistent way during unperturbed tumor growth. Further, when a parallel sorting strategy was pursued to generate scRNA-seq analysis of tumor myeloid cells from the spontaneous mammary carcinoma MMTV-PyMT, we found that both share populations with the identical signatures defined in Figure 1, albeit in different proportions, and also show a lack of co-association between "M1" and "M2" signatures amongst the clusters (Fig 3B-C, S3B-E).

While myeloid cell populations appeared to be largely defined by differentiation stage and activation programs, we considered whether other core cellular features could help to further distinguish subsets across diverse microenvironments. It is now increasingly appreciated that metabolic reprogramming accompanies differentiation of immune cells, including macrophages ${ }^{56}$. Indeed, assessment of metabolism-related genes ${ }^{57}$ demonstrated that glycolysis-associated genes were specifically enriched in the stress-responsive cell cluster whereas genes pertaining to oxidative phosphorylation were specifically enriched in $\mathrm{C}_{1 q \mathrm{q}^{+}}$ TAMs in two distinct mouse models (Fig. 3D-E, S3F). This suggests that these populations have additional important biological features in common-namely those coupled to distinct bioenergetic processes and demands.

Together, our data provides compelling evidence that "M1" and "M2" pathways have limited use in defining in vivo tumor myeloid cell differentiation and subset plasticity during normal tumor development. Rather, common microenvironmentally-induced programs and associated metabolic programs may yield greater insight in efforts to transcriptionally define and selectively target monocyte/TAM subsets.

\section{Human renal cell carcinoma-infiltrating monocytes and macrophages mirror murine populations}


We then assessed how these mouse monocyte/macrophage transcriptional programs might compare to those from human kidney cancers, which are described to have substantial myeloid cell infiltration ${ }^{58}$. We performed scRNA-seq analysis on HLA-DR ${ }^{\text {dim/+ }}$ Lin $^{-}$myeloid cells sorted from a renal cell carcinoma (RCC) sample (Fig. 4A-B, S4A). Signatures derived from previously described blood myeloid cell populations ${ }^{59}$ guided cluster identification and exclusion of cDC (Fig. S4B). Analysis of the CSF1 $R^{+} M A F B^{+}$clusters revealed a heterogenous collection of monocytes and macrophages with varying levels of CD14 and CD16 (Fig 4C-D).

As in mouse models, we detected early-stage $C D 14^{+} S 100 A 8^{+}$classical monocytes (Cluster 1) along with terminally-differentiated C1QC ${ }^{+}$TAMs (Cluster 3) (Fig. 4 E-F, Table S2). Another population (Cluster 0) were $C D 14^{+}$and differentially expressed LYPD3 and MHC-II genes, consistent with intermediate differentiation of monocytes towards TAM (“Mono-Int”; Fig. 4D-F). A population of $C D 16^{+}$non-classical monocytes (Cluster 2) also expressed IFNstimulated genes and thus appear to functionally represent 'IFN-responsive' cells (Fig. 4D-F, S4C). Finally, we found that there were a mix of cells on the monocyte-macrophage trajectory that expressed the stress-responsive program identified in mice (Fig. S4C). Of these was a cluster transcriptionally similar to TAMs but marked by high expression of the antioxidant factor SEPP1 ${ }^{60-62}$ (Cluster 4). When compared further to $C 1 Q^{+}$TAMs, this SEPP1+ cluster was less mature based on higher expression of monocytic markers (i.e. S100A genes) and lower expression of MHC-II-related genes (Fig. 4E, S4D). Pseudotime analysis of human myeloid cells recapitulated the alignment of stress- and IFN-responsive programs over the monocyte-tomacrophage trajectory (Fig. 4G), although in this RCC sample, IFN-responsive monocytes appeared more advanced in differentiation stage. As in the mouse samples, there was broad co-expression of "M1"- and "M2"-associated genes across the populations (Fig. S4E). Also, as in mice, there was a striking enrichment in a glycolytic signature ${ }^{57}$ within the stress-responsive 
$\left(\mathrm{SEPP} 1^{+}\right)$cluster as compared to the $\mathrm{C} 1 \mathrm{q}$ clusters, supporting that these cells were functional orthologs in the two species (Fig 4H). Altogether, these data confirm the limitation of "M1" and "M2" applicability in human tumors and illustrate the ability of other pathways to better define myeloid cell subsets in vivo.

\section{$\mathbf{T}_{\text {reg }}$ depletion impairs monocyte-to-macrophage differentiation and elicits inflammatory monocyte programs}

Myeloid cell density can vary across patients ${ }^{58}$, but how myeloid cell infiltration and differentiation is collectively regulated in human cancer is still not well understood. When we quantified myeloid cell populations in RCC patient biopsies using flow cytometry we found that the proportion of myeloid cells amongst live immune cells was increased in tumors of greater size and later stages (Fig. S5A). Closer examination revealed that the ratio of macrophages-tomonocytes was also specifically increased in more advanced tumors (Fig. 5A, top). This suggested that the balance between monocytes and macrophages is dynamically regulated and that tumor growth was tied to higher macrophage density.

We thus sought other immunosuppressive parameters that might work in concert with increased macrophage accumulation. $T_{\text {regs }}$ are a potent immunosuppressive force in the TIME and ablation can result in tumor clearance ${ }^{39,63}$. Interestingly, we found that $T_{\text {regs }}$ similarly accumulated as kidney tumor size increased (Fig. 5A, bottom), and that $T_{\text {reg }}$ abundance correlated well with macrophage-to-monocyte ratios in kidney as well as melanoma cancers (Fig. 5B). The positive correlation between $\mathrm{T}_{\text {reg }}$ and macrophage density spurred us to ask whether one caused the other. Using Foxp3-DTR mice, we found that depletion of $\mathrm{T}_{\text {regs }}$ dramatically reduced the macrophage-to-monocyte ratio in mouse B16 tumors (Fig. 5C, top) as 
assessed by flow cytometry. This result was phenocopied by treatment of mice with an antiCTLA-4 antibody that specifically depletes tumor $\mathrm{T}_{\text {regs }}{ }^{39}$ (Fig. 5C, bottom, S5B-C).

To further examine how $\mathrm{T}_{\text {regs }}$ may be influencing monocyte and macrophage proportions, we performed scRNA-seq analysis on mouse tumor myeloid cells from B16 tumor-bearing control and FoxP3-DTR mice. Csf1 ${ }^{+} \mathrm{Mafb}^{+}$clusters from this experiment were aggregated with those from the original wild-type B16 tumor sample in Figure 1 and we observed similar cell populations across both experiments and treatment conditions (Fig. 5D, S5D-E). Cluster

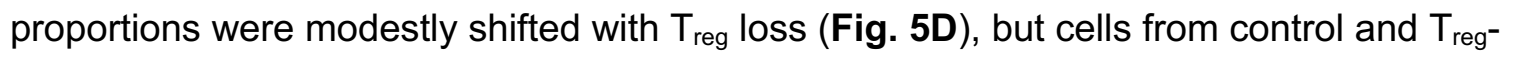
depleted tumors shared similar differentiation trajectories (Fig. 5E). However, Monocle analysis revealed differences in the accumulation of cells along the trajectory. Namely, while tumor monocytes, "Mono-Int", and TAMs from the control sample acquired progressively increased pseudotime scores, "Mono-Int", and TAM populations in the Foxp3-DTR sample did not exhibit sequential increases in pseudotime scores (Fig. 5E). In effect, TAM progression appeared stunted following depletion of $T_{\text {regs. }}$

Indeed, in addition to increased expression of inflammatory and immunomodulatory genes (e.g., Ccl24, Arg1, Retnla, Mmp12, Mmp13, Nos2), expression of monocyte-associated

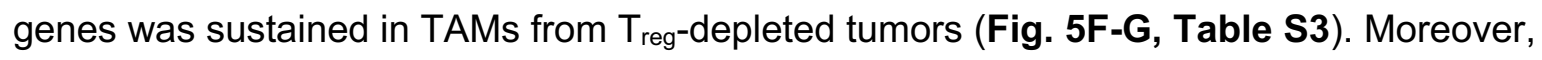
expression of genes tied to macrophage differentiation (e.g., C1qa, H2-Ab1, Apoe, Ms4a7) were decreased across stages of differentiation (Fig. 5H, S5F), further indicating these TAMs were

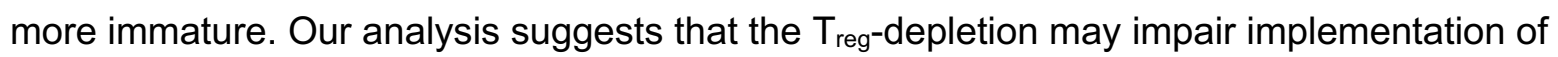
TAM transcriptional programs, a remodeling detected early during tumor monocyte differentiation. Altogether these findings support a model in which $\mathrm{T}_{\text {reg }}$ abundance promotes an accumulation of terminally-differentiated TAMs in both mouse and human tumors. 


\section{Multiparametric analysis of myeloid cell composition improves classification of kidney cancer patient antitumor responses}

Given this association between T cell subset density and TAM maturation, we sought to further explore how features of tumor macrophage infiltration could be harnessed to reliably inform features of patient outcome, such as survival. Analysis of TCGA kidney cancer samples using a myeloid gene signature from CiberSort ${ }^{64}$ demonstrated that patients with varying levels of overall myeloid cell density did not significantly differ in their survival (Fig. 6A, left). We next stratified TCGA patients based on levels of monocyte/macrophage lineage genes CSF1R and $M A F B$, finding that patients with higher levels of these appreciated only modest improvements in outcome (Fig. 6A, middle). As these genes are not strictly macrophage-specific, we leveraged our scRNA-seq analyses of human kidney samples to generate signature scores based on the ratio between macrophage and monocyte (Fig 4E). However, no significant differences in survival were revealed using this metric (Fig. 6A, right).

As TAM density did not appear to robustly inform patient outcome, we sought to test how TAM abundance corresponds with other immune parameters and may stratify patients. We thus analyzed a cohort of kidney cancer patient biopsies by flow cytometry and quantified immune cell population frequencies. Unbiased clustering analysis of samples revealed three groups of patients that exhibited distinct immune composition patterns (Fig. 6B). As suggested from our previous analysis (Fig. 5A) macrophage/monocyte ratio and $\mathrm{T}_{\text {reg }}$ density represented a strong classifier which delineated the patients and revealed a group that was highly enriched for both macrophages and $\mathrm{T}_{\text {regs }}(\mathrm{Treg}-\mathrm{Mp} ;$ red). Although the other groups were generally TAM/monocyte ${ }^{\text {lo/int }}$, one group (CD8-Mo-CDC1; pink), but not the other (CD4-cDC2; yellow), was distinguished by notable infiltration of $\mathrm{CDC} 1$, which are critical for $\mathrm{CD} 8^{+} \mathrm{T}$ cell responses $37,38,40$, and that group presented with uniformly high $\mathrm{CD}^{+} \mathrm{T}$ cell infiltration. Further, the same 
patients clustered together based on expression levels of checkpoint regulators (i.e., PD-1, CTLA-4, CD38) and proliferative capacity (i.e. Ki-67) within the CD8 ${ }^{+}$T cell compartment (Fig. 6C). Notably, $\mathrm{CD}^{+} \mathrm{T}$ cells from the CD8-Mo-cDC1 group (pink) expressed low levels of exhaustion markers PD-1 and CD38 (Fig. 6C-D) and were also distinguished by higher CTLA-4 protein expression, which may indicate ongoing activation ${ }^{65}$ (Fig. 6C-D). In contrast, the Treg$\mathrm{Mp}(\mathrm{red})$ group showed the highest levels of both exhaustion markers and Ki-67.

In testament to the heightened antitumor $\mathrm{CD}^{+} \mathrm{T}$ cell profile associated with low macrophage and $\mathrm{T}_{\text {reg }}$ abundance but high $\mathrm{CDC} 1$ density, the subset of patients with these attributes (pink) appreciated dramatically improved survival (Fig. 6E). Moreover, we found that measuring the ratio of $\mathrm{cDC} 1 \mathrm{~s}$ to macrophages through combined gene signatures rather than use of either signature alone (Fig. S6A-C) allowed for identification of kidney cancer patients with better survival in an external bulk RNAseq dataset (TCGA). Thus, fine-tuned stratification of kidney cancer TIME provided the resolution critical for identifying distinct patient classes including this CD8-Mo-cDC1 group, which defines patient with the best anti-tumor immune response regardless of tumor stage and treatment. 


\section{Discussion}

Here we undertook scRNA-seq analysis of tumor monocytes and macrophages to determine the key hallmarks of their transcriptional diversity. We found two types of differentiation to manifest during tumor development. On the one hand we found a classical lineage differentiation trajectory that progresses from monocytes-to-macrophages in a way that has been long appreciated ${ }^{66}$ with a discernable 'intermediate' monocyte ("Mono-Int”) cell population. A "Mono-Int" population is, for reference, well-described in other settings. For example, Randolph and colleagues detect 'intermediate' monocytes in lymphoid and nonlymphoid tissue in steady-state conditions ${ }^{67}$, and fluorescent real-time lineage tracing identifies cells undergoing that transition during allergic challenge ${ }^{68}$.

On the other hand, we found two differentiation layers - 'stress-responsive' and 'IFNresponsive' - that co-exist along that trajectory and that were shared across multiple mouse models as well as a profiled human kidney cancer biopsy (Fig. 1, 4). These programs were also present in other recently published studies ${ }^{18,60,69}$. For example, in a pan-cancer study, Cheng et al. discern myeloid populations whose primary distinction is their expression of IFN-induced genes (e.g., ISG15 TAMs). A notable difference in our interpretation compared to these reports lies in our incorporation of these layers within the monocyte-macrophage differentiation axis, rather than proposing them as a unique trajectory. Through independent profiling of purified monocytes and macrophages in our study and pseudotime analysis (Fig. 1-2), we find the stress-responsive signatures evident in both cell populations and indeed across them. In additional support of such a view, we found that an IFN-responsive signature was enriched amongst monocytes in one mouse model and macrophages in another (Fig. 1, 3). We believe that this represents that macrophages can differentiate in two dimensions - progression through the classical lineage as well as acquisition of specialized states characterized by examples of 
IFN or stress exposure. Intuitively, this is similar to $\mathrm{CD}^{+} \mathrm{T}$ cells that can differentiate along a naïve-effector-memory axis while also being able to layer on Th1/Th2/Th17 programs ${ }^{70}$.

Despite the latter comparison, we notably do not find any populations, nor indeed any cells, that have an exclusively "M1" or "M2" signature (Fig. 3). Individual genes such as Arg1 are associated with certain clusters, as some have observed ${ }^{18}$, but both correlation and signature analyses fail to identify any of the described 'M1' or 'M2' genes as either being selectively linked with one another in single cells, or as key classifiers of cell clusters. To this extent, the 'M1/M2' nomenclature has provided direction in the fruitful study of myeloid cell signaling and differentiation but does not appear to be accurately categorize distinct differentiation states, at least for tumors in vivo. We note the absence of data to the contrary of this conclusion in other recent reports ${ }^{55,58,69}$ although of course individual nomenclature (e.g., "M2-like") is clearly a matter of choice and needs only discussion as to which part of the in vitro signature might be biologically relevant.

One important aspect of myeloid biology that requires further elaboration is how to identify IFN- and stress-responsive phenotypes. For example, Gubin et al. use iNOS as a marker by flow cytometry to define the IFN-stimulated population induced by ICB whereas Cheng et al. utilize ISG15. Particularly in the former study which studied macrophage identity post checkpoint blockade therapies, varied levels of type I and II IFNs may also modulate properties of this differentiation layer. In the case of 'stress-responsive' populations, our data also point to IL-7R $\alpha$ expression, which may indicate involvement of TSLP signaling through heterodimeric pairing with TSLPR ${ }^{71,72}$. In human macrophages, SEPP1 was present in our study as well as in Cheng et al., but this or its homolog is not formative for what appears to be the analogous population in mice. An important set of conserved genes for 'stress-responsive' macrophages, taken from our manuscript, is their consistent and significant enrichment for 
glycolytic genes, particularly in comparison to conventional C1q 'mature' TAMs. Given that HIF1 is known to induce glycolytic genes in hypoxic conditions ${ }^{73}$, this finding raises the questions of whether these cells are selected for hypoxic environments where oxidative phosphorylation may not proceed, as well as their specific function. Going forward, the use of multiplexed imaging technologies such ion beam imaging (MIBI) ${ }^{74,75}$ and single-cell spatial transcriptomics ${ }^{76-78}$ will enable this analysis.

Our investigation of monocyte/macrophage differentiation led us to explore how its regulation could inform our understanding of antitumor immunity. Analysis of kidney cancer and melanoma patient cohorts revealed an increase in macrophage-to-monocyte ratios with tumor grade, a rise that coincided with $T_{\text {reg }}$ density and was $T_{\text {reg-dependent. }} \mathrm{T}_{\text {regs }}$ exert potent immunosuppression and are thought to restrain T cell activity and antitumor responses through modulation of DC stimulatory capacity, production of immunosuppressive cytokines and substrates, and competitive usage of growth factors and metabolic byproducts ${ }^{39,79-81}$. It is becoming clear now that tumor $\mathrm{T}_{\text {regs }}$ also strongly influence the monocyte/macrophage lineage, likely through multiple mechanisms. In a recent study, tumor $\mathrm{T}_{\text {regs }}$ promoted tumor macrophage numbers by supporting their mitochondrial capacity and viability ${ }^{82}$. Here our scRNA-seq data demonstrates that early-stage monocytes and "Mono-Int" cells are already unable to properly implement TAM-associated transcriptional programs in the absence of $T_{\text {regs }}$, indicating that $T_{\text {regs }}$ also fuel macrophage differentiation processes. This liaison between $T_{\text {regs }}$ and macrophages mirrors one identified elsewhere such as adipose fat of lean mice where $T_{\text {regs }}$ are thought to actively maintain homeostasis and hold inflammatory macrophages at bay ${ }^{83,84}$. Similarly, during the resolution of injury and inflammation in skeletal muscle and heart tissue, a transition from pro- to anti-inflammatory macrophages occurs in a manner that appears to rely on $\mathrm{T}_{\text {reg }}$ accumulation $^{85-88}$. That $\mathrm{T}_{\text {regs }}$ may act on tumor macrophages in a similar fashion offers another 
example of how the TIME can exploit immune programs of "accommodation" that are otherwise in place to achieve tissue homeostasis in the face of perturbations ${ }^{89}$.

Accumulation of a broad swath macrophages in the TIME has previously been implicated with poor outcome ${ }^{90}$. Consistent with this but at higher resolution, we detected a group of kidney cancer patients for whom high macrophage-to-monocyte abundance was associated with diminished T cell infiltration and exhaustion of those cells detected, concurring with other very recent reports ${ }^{58,60}$. Our manuscript thus points to an emerging trio of $T_{\text {regs, }}$, macrophages, and exhausted T cells, whereby effector T cells may be corrupted through direct cellular interactions with TAMs, as has been suggested by observations of TAM-CD8 ${ }^{+} \mathrm{T}_{\text {cell }}$ colocalization in $\mathrm{cCRCC}^{60}$, or indirectly through macrophage-induced $\mathrm{T}_{\text {reg }}$ expansion and activity $^{15,91}$ or DC suppression ${ }^{39,92}$.

Yet, high myeloid infiltration or skewed macrophage-to-monocyte ratios alone were not prognostic for KIRC patient survival. Indeed, although macrophages have often been found to be negatively associated with patient outcome, macrophage abundance as a sole biomarker has not been universally useful with prior studies similarly reporting instances in which macrophage abundance is not informative for patient cohorts with specific cancer sub-types, treatment regimens, or tumor stage ${ }^{93-96}$. Clustering analysis of kidney TIME composition using comprehensive immune parameters, however, uncovered an archetype characterized by low macrophage-to-monocyte in conjunction with high cDC1 infiltration. These patients (CD8-MocDC1) had elevated infiltration of $C D 8^{+} \mathrm{T}$ cells with low surface expression of proteins associated with exhaustion and appreciated highly enhanced survival rates (Fig. 6, pink). Notably, recent work focused on ascertaining the different immune archetypes across solid tumors suggests that these patient groups span cancer type ${ }^{51}$. 
Identification of a CD8-Mo-cDC1 archetype emphasizes the value of integrating multiparametric biomarkers as a means to better parse patient outcome and to establish principles of TIME organization. Given that T cell activity appears to be collectively influenced by multiple immune cell populations with distinct partnering patterns, our analysis suggests that dual targeting of TIME axes may elicit the best CD8+ T cell responses. For example, reprogramming and/or depletion of macrophages may relieve active suppression ${ }^{5,44}$ and strategies that boost $\mathrm{CDC} 1$ recruitment and survival ${ }^{2}$ may further benefit even those with favorable macrophage-to-monocyte density. It is also notable that this protective archetype is specifically enriched for monocytes. Indeed, monocyte differentiation into macrophages may not be inevitable and accumulation of "Mono-Int" cells have been detected in multiple forms of inflammation ${ }^{17,29,97-100}$. Additionally, the potential importance of monocytes is indicated by their increased numbers in the blood of ICB responsive as compared to non-responsive melanoma patients $^{50}$. In ccRCC patients, IFN-responsive TAMs exhibited lower levels of HLA-DR, reminiscent of "Mono-Int" cells described here, and higher levels of these TAM-ISG ${ }^{\text {hi }}$ were predictive of survival after TKI treatment ${ }^{94}$. Such a relationship opens questions across cancer type; namely, whether "Mono-Int" are distinct in their antitumor function, and how might monocytes be additive or synergistic with $\mathrm{CDC} 1$ to drive antitumor CD8 T cells?

Altogether these findings contribute to the endeavor of clarifying useful distinctions in myeloid gene expression and highlights settings in which multiparametric analysis of tumor myeloid composition can inform patient immune archetype and guide development of relevant therapies. 


\section{Methods}

\section{Mice}

The following mice were housed and/or bred under specific pathogen-free conditions at the University of California, San Francisco Animal Barrier Facility: C57BL/6J (The Jackson Laboratory), MMTV-PyMT-mCherry-OVA transgenic ${ }^{101}$, and Foxp3-DTR (The Jackson Laboratory). All mice were handled in accordance with NIH and American Association of Laboratory Animal Care standards, and experiments were approved by the Institutional Animal Care and Use Committee of the University of California, San Francisco.

\section{Human Tumor Samples}

$\mathrm{RCC}$ or melanoma tumor samples were transported from various cancer operating rooms or outpatient clinics. All patients consented by the UCSF IPI clinical coordinator group for tissue collection under a UCSF IRB approved protocol (UCSF IRB\# 20-31740). Samples were obtained after surgical excision with biopsies taken by Pathology Assistants to confirm the presence of tumor cells. Patients were selected without regard to prior treatment. Freshly resected samples were placed in ice-cold DPBS or Leibovitz's L-15 medium in a $50 \mathrm{~mL}$ conical tube and immediately transported to the laboratory for sample labeling and processing. The whole tissue underwent digestion and processing to generate a single-cell suspension. In the event that part of the tissue was sliced and preserved for imaging analysis, the remaining portion of the tissue sample was used for flow cytometry analysis.

\section{Tumor Cell Lines}

B16-F10 cells (ATCC, CRL-6475) were purchased and cultured at $37^{\circ} \mathrm{C}$ in $5 \% \mathrm{CO}_{2}$ in DMEM (Invitrogen), 10\% FCS (Benchmark), Penicillin, Streptomycin, and L-Glutamine (Invitrogen). B16-F10-ZsGreen was previously generated in our laboratory as described ${ }^{102}$. 


\section{Mouse Tumor Cell Injections and Growth}

Prior to injection, adherent B16-F10 or B16-ZsGreen tumor cells were dissociated with $0.05 \%$ Trypsin-EDTA (Thermo Fisher Scientific) and washed 2-3X with DPBS (Thermo Fisher Scientific). $1.0 \times 10^{5}-2.5 \times 10^{5}$ cells were resuspended in DPBS and mixed 1:1 with Matrigel GFR (Corning). Mice were injected subcutaneously with a volume of $50 \mu$ leither unilaterally or bilaterally depending on the experimental setup. Tumor tissue was harvested approximately $12-$ 16 days later.

MMTV-PyMT-mCherry-OVA transgenic mice were bred and genotyped for the transgene by PCR. Spontaneous tumor growth was monitored, and tumors were harvested when the mice were approximately 20-30 weeks of age.

\section{Mouse Tissue Processing and Flow Cytometry Staining}

Mouse tumor tissue was harvested and enzymatically digested with $0.2 \mathrm{mg} / \mathrm{ml}$ DNase I (SigmaAldrich), 100U/ml Collagenase I (Worthington Biochemical), and 500U/ml Collagenase Type IV (Worthington Biochemical) for 30 minutes at $37^{\circ} \mathrm{C}$ while under constant agitation. Blood was collected via cardiac puncture from mice that were euthanized by overdose with $2.5 \%$ Avertin. Blood samples were treated with $175 \mathrm{mM} \mathrm{NH}_{4} \mathrm{Cl}$ for 5 minutes at room temperature to lyse red blood cells.

Samples were filtered, washed with stain media (DPBS, 2\% FCS), and resuspended again with stain media. Cells from this single cell suspension were washed with DPBS and stained with Zombie NIR fixable viability dye (BioLegend) for 30 minutes at $4^{\circ} \mathrm{C}$. Cells were washed and resuspended with stain media containing anti-CD16/32 (BioXCell), 2\% rat serum, $2 \%$ Armenian hamster serum, and antibodies against surface proteins of interest. Cells were stained for 30 minutes at $4^{\circ} \mathrm{C}$. At times cells were then washed and stained for intracellular 
protein levels, for which they were fixed, permeabilized, and stained according to BD Cytofix/Cytoperm Kit (BD Biosciences) or the FoxP3/Transcription Factor Staining Buffer Set (Thermo Fisher).

The following antibodies were from Biolegend: anti-mouse CD45, anti-mouse Ly-6C (HK1.4), anti-mouse CD11b (M1/70), anti-mouse CD11c, anti-mouse MHC-II, anti-mouse F4/80, anti-mouse CD24, anti-mouse Ly-6G, anti-mouse NK1.1, anti-mouse CD90.2, antimouse/human CD45R/B220, anti-mouse CD301b, anti-mouse CD64, anti-mouse CD127. The following antibodies were from BD Biosciences: anti-mouse Siglec-F, anti-mouse CD106. The following antibodies were from R\&D: anti-mouse/human ARG1, normal sheep IgG. The following antibodies were from ThermoFisher: anti-mouse FoxP3.

Following staining, cells were washed, resuspended in stain media, and analyzed on a BD Biosciences Fortessa or sorted with a BD Biosciences FACSAria Fusion. Flow cytometry data was analyzed using FlowJo software (BD Biosciences).

\section{Human Tissue Processing and Flow Cytometry Staining}

Tumor or metastatic tissue was thoroughly chopped with surgical scissors and transferred to gentleMACS C Tubes (Miltenyi Biotec) containing $20 \mathrm{uL} / \mathrm{mL}$ Liberase TL (5 mg/ml, Roche) and $50 \mathrm{U} / \mathrm{ml}$ DNAse I (Roche) in RPMI 1640 (Invitrogen) per $0.3 \mathrm{~g}$ tissue. gentleMACS C Tubes were installed onto the gentleMACS Octo Dissociator (Miltenyi Biotec) and incubated for 45 minutes according to the manufacturer's instructions. Samples were then quenched with $15 \mathrm{~mL}$ of sort buffer (DPBS, 2\% FCS, 2mM EDTA), filtered through $100 \mu \mathrm{m}$ filters, and spun down. Red blood cell lysis was performed with $175 \mathrm{mM}$ ammonium chloride if needed.

Cells were incubated with Human FcX (Biolegend) to prevent non-specific antibody binding. Cells were then washed in DPBS and incubated with Zombie Aqua Fixable Viability Dye (Thermo). Following viability dye, cells were washed with sort buffer and incubated with cell surface antibodies that had been diluted in the BV stain buffer (BD Biosciences) for 30 minutes 
on ice. Cells were subsequently fixed in either Fixation Buffer (BD Biosciences) or in Foxp3/Transcription Factor Staining Buffer Set (eBioscience) if intracellular staining was required. The following antibodies were from BD Biosciences: anti-human HLA-DR, anti-human CD56, anti-human CD127, anti-human CD25, anti-human CD45RO, anti-human PD-1, antihuman CTLA-4, and anti-human CD64. The following antibodies were from ThermoFisher: antihuman $\mathrm{CD} 45$, anti-human $\mathrm{CD} 3 \varepsilon$, anti-human FoxP3, anti-human Ki-67, anti-human CD19, antihuman CD20, anti-human CD56, and anti-human CD11c. The following antibodies were from Biolegend: anti-human CD4, anti-human CD8 $\alpha$, anti-human CD38, anti-human CD16, CD1C/BDCA-1, anti-human CD14, anti-human CD304, and streptavidin. Anti-human BDCA-3 was purchased from Miltenyi,

Stained cells were washed and analyzed on a BD Biosciences Fortessa or sorted with a BD Biosciences FACSAria Fusion. Flow cytometry data was analyzed using FlowJo software (BD Biosciences).

\section{Single Cell RNA-Sequencing Data Generation}

Sorted cells were resuspended at a concentration of $1 \times 10^{3}$ cells $/ \mu$ in media (DPBS, $0.04 \%$ BSA) and loaded onto the Chromium Controller (10X Genomics). Samples underwent singlecell encapsulation and cDNA library preparation using the Chromium Single Cell 3' v1 or v2 Reagent Kits (10X Genomics). The cDNA library was sequenced on an Ilumina HiSeq 4000 (Illumina).

\section{Single Cell RNA-Sequencing Data Processing}

Sequencing data was processed using 10X Genomics Cell Ranger V1.2 pipeline. Fastq files were generated from llumina bcl files with the Cell Ranger subroutine mkfastq. Fastq files were then processed with Cell Ranger's count to align RNA reads against UCSC mm10 or GRCh38 
genomics for mouse and human cells, respectively, using the aligner STAR ${ }^{103}$. Redundant unique molecular identifiers (UMI) were filtered, and a gene-cell barcode matrix was generated with count. Mkfastq and count were run with default parameters.

For mouse B16 tumor samples, the gene-cell barcode matrix was passed to the $\mathrm{R}$ software package Seurat (v2.3.0) $)^{104}$ for all downstream analyses. Genes that were expressed in at least 3 cells were included. Cells that did not express at least 200 genes were excluded, as were those that contained $>5 \%$ reads associated with cell cycle genes ${ }^{105,106}$. For mouse PyMT and human RCC tumor samples, raw feature-barcode matrices were loaded into Seurat $(\mathrm{v} 3.1 .5)^{107}$ and genes with fewer than 3 UMls were dropped from the analyses. Matrices were further filtered to remove events with greater than $20 \%$ percent mitochondrial content, events with greater than $50 \%$ ribosomal content, or events with fewer than 200 total genes. The cell cycle state of each cell was assessed using a published set of genes associated with various stages of human mitosis ${ }^{108}$.

Using Seurat's ScaleData function, read counts were log2 transformed and scaled using each cell's proportion of cell cycle genes as a nuisance factor. A set of highly variable genes was generated by Seurat's FindVariableGenes function, which were used for principal component (PC) analysis. Genes associated with PCs (selected following visualization with scree plots) were used for graph-based cluster identification and dimensionality reduction using t-distributed stochastic neighbor embedding ( $t$-SNE) analysis. Seurat's FindAllMarkers function was used for subsequent cluster-based analyses, including cluster marker identification and DE gene analyses.

\section{Single Cell RNA-Sequencing Signature Generation}

To generate mouse monocyte- and macrophage-specific gene signatures (Fig. 1), sorted monocyte, TAM1, and TAM2 samples were aggregated, log2 transformed, and scaled using Seurat. DE gene analysis was performed using FindMarkers with the parameters $\log N$ fold 
change $>1.5$ and a min.pct of 0.25 . Genes were selected by ranked fold change and the criteria that $\min$. pct $1 / \min$. pct2 $>1.5$. Genes used for cell cycle regression analysis were excluded. The top 10 genes (or fewer if less remained) were median normalized and aggregated, scaled 0-1, and plotted on specific $t$-SNE plots.

Gene signatures for cellular programs such as metabolism", "M1" and "M2" polarization $^{55}$, and MHC-II-associated genes (GSEA, REACTOME_MHC_CLASS_II_ANTIGEN_PRESENTATION), previously published cell types $^{53,59}$, or cell populations identified here were generated by taking the mean of lognormalized expression for a particular set of genes related to the specific pathway or phenotype. To visualize the distribution of these scores across cells, we binarized the distribution of the score at the 70th percentile unless specified otherwise and overlaid on the calculated $t$-SNE coordinates.

For correlation analysis of "M1" and "M2" genes, the expression of each gene in the signatures was calculated for each B16 tumor $\mathrm{Csf1r}^{+} \mathrm{Mafb}^{+}$cluster cell and binarized at the median. A cross-correlation gene-gene matrix was obtained using the $\mathrm{R}$ cor function with method="pearson".

\section{Single Cell RNA-Sequencing Sample Aggregation}

To perform pairwise comparison analyses between B16 tumor myeloid cell clusters from

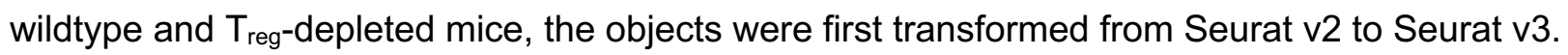
The raw UMI counts were renormalized using person residuals from "regularized negative binomial regression," with sequencing depth a covariate in a generalized linear model via the $R$ sctransform package ${ }^{109}$. Pairwise "anchor" cells were identified between the three objects using the wild-type mouse from Figure 1 as a reference via the Seurat FindIntegrationAnchors function. Briefly, each pair of samples was reduced to a lower dimensional space using 
diagonalized Canonical Correlation Analysis (CCA) using the top 3000 genes with the highest dispersions. The canonical correlation vectors were normalized using L2-normalization. Multiple Nearest Neighbors (MNNs) across datasets were identified for each cell in each dataset and cell-cell similarities are used as anchors to integrate the datasets together using the Seurat IntegrateData function.

\section{Single Cell RNA-Sequencing Pseudotime Analysis}

Raw UMI counts from the cleaned and processed Seurat objects for the control and $\mathrm{T}_{\text {reg}}{ }^{-}$ depleted mouse experiment were extracted and coerced into Monocle $2^{54,110}$. CellDataSet objects, normalizing the data using a negative binomial distribution with fixed variance (negbinom.size). Each object was independently processed to identify a pseudotime trajectory. Briefly, each object was processed to estimate per-cell coverage and sequencing depth (estimateSizeFactors) and gene dispersions (estimateDispersions). Cells were further filtered to retain high-quality cells with $>=500$ genes and genes were filtered to retain only those in at least 10 cells. The dataset was reduced to 2 dimensions using the DDRTree algorithm and the marker genes that differentiated the $L y 6 c 2^{+} \mathrm{Hp}^{+}$monocytes and $C 1 q a^{+} \mathrm{TAM}$ clusters from other cell types were used to guide the trajectory inference. Relative pseudotime was obtained through a linear transformation relative to the cells with the lowest and highest pseudotimes (1min_pseudotime)/max_pseudotime. The "wave" plots in Figure 1 were constructed using the Seurat LogNormalized counts and the relative pseudotime described above for the top 5 genes per cluster as identified by scRNA-seq.

\section{TCGA Survival Analyses}

Tumor RNAseq counts and transcripts-per-million (TPM) values for kidney renal clear cell carcinoma (KIRC) samples from the Toil recompute data in the TCGA Pan-Cancer (PANCAN) cohort $^{111}$ were downloaded from the UCSC Xena browser ${ }^{112}$. A gene signature score for each 
patient was calculated using the gene signature score method below. The feature gene signature scores were calculated using an $\mathrm{m} \times \mathrm{n}$ matrix where $\mathrm{m}$ represented the TPM normalized log2 counts per million (logCPM) expression of the feature signature genes and $n$ represented the selected sample set ${ }^{113}$. The expression of each gene was converted to percentile ranks across the samples using the SciPy Python module ${ }^{114}$. The top and bottom 30 percentile were then used to define low and high patients for each respective signature unless otherwise noted.

\section{In Vivo Mouse Treatments}

To deplete $\mathrm{T}_{\text {reg }}$ cells, Foxp3-DTR and control mice were injected intraperitoneally with 500ng of unnicked diptheria toxin (List Biologics, 150). Mice were typically injected 9, 10, and 12 days following initial inoculation with tumor cells.

For specified experiments, wild-type mice were injected intraperitoneally 7, 9, 10, 11, and 13 days following tumor injection with $250 \mu \mathrm{g}$ of anti-mouse CTLA-4 IgG2c (modified clone 9D9, Bristol-Myers-Squibb), mouse IgG2C isotype, anti-mouse CTLA-4 IgG1 (modified clone 9D9, Bristol-Myers-Squibb), or mouse IgG1 isotype.

\section{Statistical analysis and data visualization}

Comparisons between groups were analyzed using GraphPad Prism software. Experimental group allocation was determined by genotype or by random designation when all wild-type mice were used. Error bars represent mean \pm SEM calculated with Prism unless otherwise noted. Comparisons between 2 groups were analyzed with Student's $t$-test. For statistical measures between more than 2 groups, one-way ANOVA was performed unless otherwise noted. Nonsignificant comparisons are not shown. Investigators were not blinded to experiment group assignment during experimental data generation or analyses. The R packages Seurat and ggplot2 were used to generate figures. 


\section{Acknowledgments}

We would like to thank E. Wan and the Institute for Human Genetics at UCSF for helping prepare samples for scRNA-seq as well as the UCSF Flow Cytometry Core for maintenance of flow cytometers and sorters. We would like to also thank J.J. Engelhardt and Bristol-MyersSquibb for Fc-modified anti-CTLA-4 antibodies. This work was supported by the US National Institutes of Health (R01CA197363 and U01CA217864 to M.F.K). A.M.M. is supported by the Cancer Research Institute as a Cancer Research Institute/Amgen Fellow. Acquisition and analysis of certain human samples was partially funded by contributions from Abbvie, Amgen, Bristol-Myers Squibb, Genentech, and Pfizer.

\section{Author Contributions}

A.M.M, A.J.C., M.B., and M.F.K. designed experiments. A.M.M, A.J.C., and M.B. performed experiments unless specified. A.R.R. and J.L.P. participated in the processing and analysis of scRNA-seq. B.S. participated in the analysis of TCGA samples. J.T., A.B., R.J.A., M.K.R, and K.C.B. participated in experimental data collection and flow cytometry analysis. V.C. managed the acquisition of human tumors samples. A.M.M., A.J.C., and M.F.K. wrote the manuscript. A.M.M., A.J.C., M.B., A.B., and R.J.A. edited the manuscript.

\section{Declaration of Interests}

M.F.K. is a founder and shareholder in Pionyr Immunotherapeutics that develops novel immunotherapeutics that target and tune myeloid cells. M.B and J.L.P. are shareholders in Pionyr Immunotherapeutics. 
Figure 1. ScRNA-seq analysis of mouse B16 tumor myeloid cells maps transcriptional heterogeneity amongst monocytes and TAMs.

(A) Schematic illustration of workflow for isolation of specified myeloid populations from B16 tumors subcutaneously implanted in wild-type C57BI/6 mice.

(B) $t$-SNE plot of graph-based clustering of $\mathrm{Ly}_{6 \mathrm{C}}{ }^{+} \mathrm{CD} 11 \mathrm{~b}^{+}$monocytes and $\mathrm{Ly}_{6 \mathrm{C}} \mathrm{MHCII}^{+}$ myeloid cells that were sorted and pooled from B16 tumors, and underwent scRNA-seq (A).

(C) Expression of Csf1r (left) and Mafb (middle) on t-SNE plot of bulk myeloid cells (B), and display of selected $\mathrm{Csf1r}^{+} \mathrm{Mafb}^{+}$clusters (right).

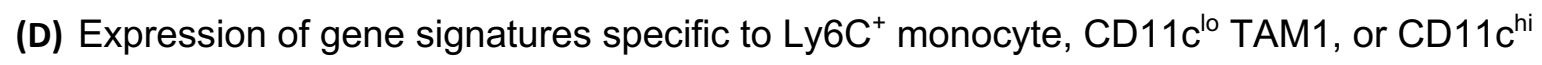
TAM2 populations (A, Fig. S1E) displayed on $t$-SNE plot of $\mathrm{Csf}_{1} \mathrm{r}^{+} \mathrm{Mafb}^{+}$myeloid cells (C). Cells with top median of signature expression level labeled in red.

(E) Heatmap displaying expression levels of top 5 differentially expressed (DE) genes between $\mathrm{Csf1r}^{+} \mathrm{Mafb}^{+}$cell clusters (C). Genes ranked by fold change.

(F) Expression levels of selected genes amongst $\mathrm{Csf1}^{+} \mathrm{Mafb}^{+}$cell clusters (C).

(G) Differentiation trajectory model using Monocle analysis of cells from Csf1r ${ }^{+} \mathrm{Mafb}^{+}$ clusters (C). Color coding corresponds to previous labels (B).

(H) Graph of relative pseudotime values of $\mathrm{Csf1r}^{+} \mathrm{Mafb}^{+}$cluster cells (C) from Monocle analysis (G).

(I) Expression levels of cluster-specific genes $(\mathbf{E})$ over relative pseudotime $(\mathbf{H})$. Each line corresponds to an individual gene.

\section{Figure 2. ScRNA-seq analysis highlights layering of microenvironment-induced} programs during tumor monocyte-to-macrophage differentiation. 
(A) $t$-SNE plot of graph-based clustering (top) of Ly6C ${ }^{+}$monocytes sorted from B16 tumors and processed for scRNA-seq (Fig. 1A), and heatmap displaying expression levels of top 5 DE genes between clusters (bottom) with genes ranked by fold change.

(B) $t$-SNE plot and graph-based clustering (top) of CD11 $\mathrm{c}^{\text {hi }}$ TAMs sorted from B16 tumors and processed for scRNA-seq (Fig 1A), and heatmap displaying expression levels of top $5 \mathrm{DE}$ genes between clusters (bottom) with genes ranked by fold change.

(C) Stress-responsive cells (Cluster 2) from bulk B16 myeloid cells (Fig. 1B) were selected for further clustering analysis (top). Heatmap of expression levels of monocyte- and macrophage-specific genes (Fig. 1E) by Cluster 2 sub-clusters (bottom).

(D) Heatmap of DE gene expression levels between Cluster 2 and Cluster 3 of bulk tumor myeloid sample (Fig. 1B). Genes ranked by degree of exclusivity to a given cluster (min.pct1/min.pct2).

(E) Expression levels of IL7R $\alpha$ and VCAM-1, as assessed by flow cytometry, of "Mono-Int" $\left(\right.$ Ly6C $\left.^{+} \mathrm{CD}^{+} 4^{+}\right)\left(\right.$top) and TAMs (Ly6C- F4/80+ $\mathrm{CD}^{+} 4^{+}$(bottom) from B16 tumors.

(F) Example (left) and quantification (right) of intracellular ARG1 expression by VCAM-1 ${ }^{+}$ (top) or IL7r $\alpha^{+}$(bottom) TAMs from B16 tumors using flow cytometry. ARG $1^{+}$gating determined by isotype control. Data are representative of 2 independent experiments with 3-5 mice (mean \pm SEM).

(G) Expression levels of selected genes along differentiated trajectory generated by Monocle (Fig. 1G).

(H) Schematic model of tumor monocyte-to-macrophage differentiation that integrates lineage-associated and microenvironmentally-induced transcriptional programs.

Figure 3. B16 and PyMT tumor monocyte/macrophage heterogeneity can be attributed to diversity in transcriptional and metabolic programs, but not "M1/M2" polarization. 
(A) Heatmap (left) and density plot (right) of Pearson r coefficient scores between "M1"and "M2"-associated gene expression levels within Csf1r ${ }^{+} \mathrm{Mafb}^{+}$cells from B16 tumors (Fig. 1C).

(B) $t$-SNE plot of $\mathrm{Csf1r}^{+} \mathrm{Mafb}^{+}$clusters from B16 tumors (top; Fig. 1C) with expression levels of "M1" (bottom, left) and "M2" (bottom, right) gene signatures (A) displayed. Cells with top median of signature expression level labeled in red.

(C) $t$-SNE plot and graph-based clustering of $\mathrm{Csf1}^{+} \mathrm{Mafb}^{+}$clusters from PyMT tumor myeloid cells that were sorted and processed for scRNA-seq (top; Supplementary Fig. 3B). Expression levels of "M1" (bottom, left) and "M2" (bottom, right) gene signatures (A) displayed. Cells with top 70 percentile of signature expression level labeled in red

(D) Expression levels of glycolysis (left) and oxidative phosphorylation (“OxPhos”) (right) gene signatures (Supplementary Fig. 3F) displayed on $t$-SNE plot of $\mathrm{Csf1r}^{+} \mathrm{Mafb}^{+}$ clusters from B16 tumors (Fig. 1C). Cells with top 70 percentile of signature expression level labeled in red

(E) Expression levels of glycolysis (left) and oxidative phosphorylation ("OxPhos") (right) gene signatures (Supplementary Fig. 3F) displayed on $t$-SNE plot of $\mathrm{Csf1r}^{+} \mathrm{Mafb}^{+}$ clusters from PyMT tumors (C). Cells with top 70 percentile of signature expression level labeled in red

\section{Figure 4. Human RCC and mouse tumor myeloid compartments exhibit shared} transcriptional features.

(A) Schematic of human RCC biopsy sample processed for scRNAseq analysis.

(B) $t$-SNE plot of graph-based clustering of bulk myeloid ( Lin $^{-} \mathrm{HLA}_{-} \mathrm{DR}{ }^{+}$) cells sorted from human RCC biopsy sample $(\mathbf{A})$. 
(C) Gene expression levels of CSF1R (left) and MAFB (right) displayed on $t$-SNE plot of human RCC-infiltrating myeloid cells (B).

(D) Expression levels of selected genes (CD14, FCGR3A, CD68) or gene signature (MHCII-associated genes) displayed on $t$-SNE plot of $\mathrm{CSF}_{1 R^{+}} \mathrm{MAFB}^{+}$clusters (C).

(E) Heatmap of top 5 DE genes expressed by CSF1R ${ }^{+}$MAFB $^{+}$clusters (C). Genes ranked by fold change.

(F) Expression levels of selected genes by $C S F 1 R^{+} M A F B^{+}$cluster cells (C).

(G) Differentiation trajectory model generated by Monocle analysis of CSF1 $R^{+} M A F B^{+}$ clusters (C).

(H) Expression levels of glycolysis-associated gene signature by cells in stress-responsive (Cluster 4$)$ and $C 1 Q^{+}$TAM (Cluster 3$)$ cells (B).

\section{Figure 5. Immunosuppressive $\mathrm{T}_{\text {reg }}$ cells promote tumor monocyte-to-macrophage differentiation.}

(A) Human RCC biopsies were measured and processed for flow cytometric analysis. The ratio of macrophage-to-monocyte $\left(\log _{2}\right)$ cell numbers (top) and $T_{\text {reg }}$ frequency amongst CD45 ${ }^{+}$cells (bottom) were quantified. ${ }^{*} p<0.05$.

(B) Dot plot and Spearman's correlation coefficient of macrophage-to-monocyte cell number ratio $\left(\log _{2}\right)$ and $\mathrm{T}_{\text {reg }}$ frequency within $\mathrm{CD} 45^{+}$cells in human $\mathrm{RCC}$ (top) and melanoma (bottom) biopsies that were analyzed by flow cytometry.

(C) Quantification of the ratio between macrophages (Ly6C- F4/80+ CD64 $^{+}$) and monocytes $\left(\right.$ Ly6C $\left.^{+} \mathrm{CD} 11 \mathrm{~b}^{+}\right)$cell number ratio in B16 tumors of DT-treated control and Foxp3-DTR mice (top), or of wild-type mice treated with depleting anti-CTLA-4 (IgG2c clone) or isotype antibody (bottom). Data is representative of 2 independent experiments. ${ }^{* *} p$ $<0.01,{ }^{* * *} p<0.0001$ 
(D) $t$-SNE plot of graph-based clustering (top) of aggregated B16-infiltrating $\mathrm{Csf1r}^{+} \mathrm{Mafb}^{+}$ cells from wildtype mice (Fig. 1), as well as DT-treated control and Foxp3-DTR mice (Supplementary Fig. 5D). Cell numbers in specified clusters were quantified (bottom).

(E) Differentiation trajectory model generated from Monocle analysis (top) and relative pseudotime values (bottom) of $\mathrm{Csf1}^{+} \mathrm{Mafb}^{+}$cluster cells from B16 tumors from DTtreated control (left) and FoxP3-DTR mice (right).

(F) Volcano plot displaying DE genes between B16 tumor "Mono-Int" (top) and C1qa ${ }^{+}$TAM (bottom) cluster cells from DT-treated control and FoxP3-DTR mice (D). Genes with > 0.4 log-fold changes and an adjusted p value of 0.05 (based on Bonferroni correction) are highlighted in red. Genes of interest labeled.

(G) Expression of selected monocyte-associated genes displayed on the differentiation trajectory (E) of control (top) or Foxp3-DTR (bottom) B16 tumor-infiltrating Csf1r $\mathrm{Mafb}^{+}$ cells.

(H) Expression of selected macrophage-associated genes displayed on the differentiation trajectory (E) of control (top) or Foxp3-DTR (bottom) B16 tumor-infiltrating Csf1r Mafb $^{+}$ cells.

Figure 6. Multiparametric analysis of tumor myeloid composition identifies kidney cancer patients with effector $\mathrm{CD}^{+} \mathrm{T}$ cell responses and improved survival rates.

(A) Survival curves of kidney tumor patients whose TCGA tumor samples exhibited high $(33 \%)$ or low (33\%) levels of expression levels of pan-myeloid gene signatures derived from CIBERSORT (left), MAFB and CSF1R (middle), or ratio of monocyte-to-TAM gene signatures (Fig. 4) (right). 
(B) Heatmap of specified immune cell population frequencies detected in human kidney tumor samples by flow cytometry.

(C) Heatmap of specified surface receptor or Ki-67 expression frequencies amongst $\mathrm{CD}^{+} \mathrm{T}$ cells from human kidney tumor samples that were analyzed with flow cytometry.

(D) Quantification of the frequency of $\mathrm{CD}^{+} \mathrm{T}$ cells from human tumor kidney samples that are $\mathrm{PD} 1^{+}$or $\mathrm{CD}_{38}{ }^{+}$. Labeling of dots corresponds to patient groups $(\mathbf{B}, \mathbf{C})$.

(E) Survival curves of kidney cancer patients in cohort analyzed (B-D).

\section{Supplementary Figure 1}

(A) Flow cytometry gating schematic for sorting and analysis of specified B16 tumor myeloid populations.

(B) Quantification of cells recovered from scRNA-seq analysis of B16 bulk tumor myeloid cells (Fig. 1B).

(C) Quantification of unique molecular identified (UMI) counts in each myeloid cell cluster (Fig. 1B).

(D) Expression of Flt3 (left) and Kit (right) displayed on t-SNE plot of B16 tumor myeloid cells (Fig. 1B).

(E) List of cell type-specific DE genes identified from comparative analysis of Ly6C ${ }^{+}$ monocyte, CD11 $\mathrm{c}^{\text {lo }}$ TAM1, or CD11 $\mathrm{c}^{\text {hi }}$ TAM2 populations that were individually sorted and processed for scRNA-seq analysis. (Fig. 1A).

(F) Expression levels of selected stress-response genes amongst $\mathrm{Csf1}^{+} \mathrm{Mafb}^{+}$cell clusters (Fig. 1C).

(G) Differentiation trajectory model of $\mathrm{Csf}^{+} \mathrm{r}^{+} \mathrm{Mafb}^{+}$cells (Fig. 1G) with display of cells from individual clusters (Fig. 1C). 
(H) Expression of selected canonical monocyte- and macrophage-related genes displayed on the differentiation trajectory plot (Fig. 1G).

Flow cytometric gating of B16 tumor-infiltrating monocytes (pink; Ly6C+ CD64), "MonoInt" (magenta; Ly6C $\mathrm{CD}^{+} 4^{+}$), and macrophages (purple; Ly6C- CD64 ${ }^{+}$) (top), and their surface protein expression of MHC-II (middle) and F4/80 (bottom),

\section{Supplementary Figure 2}

(A) $t$-SNE plot of sorted Ly6C ${ }^{+}$monocytes sorted from B16 tumors (left; Fig. 2A) with expression of $L y 6 c 2$ (middle) and $H 2-A b 1$ (right) displayed.

(B) $t$-SNE plot of graph-based clustering (left, top) and quantification (left, bottom) of CD11 $\mathrm{c}^{\mathrm{lo}}$ TAMs sorted from B16 tumors and processed for scRNA-seq, and heatmap showing expression levels of top 5 DE genes between clusters (right). Genes are ranked by fold change.

(C) $t$-SNE plot of sorted CD11 $c^{\text {hi }}$ TAMs sorted from B16 tumors (left; Fig. 2B) with expression of $L y 6 c 2$ (middle) and H2-Ab1 (right) displayed.

(D) Expression levels of Mgl2 displayed on t-SNE plot of bulk $\mathrm{Csf1r}^{+} \mathrm{Mafb}^{+}$cells sorted from B16 tumors (left; Fig. 1C), and MGL2 surface protein levels on TAMs from B16 tumors (right).

(E) $t$-SNE plot of graph-based clustering analysis of stress-responsive cluster (Cluster 2) from bulk myeloid cells (Fig. 2C).

(F) Quantification of frequency of VCAM-1 ${ }^{-}$IL7R $\alpha^{-}, \mathrm{VCAM}-1^{+}$, or IL7Ra ${ }^{+}$cells detected within B16 tumor monocyte, Mono-Int, or TAMs by flow cytometry. Data is pooled from 2 independent experiments, each with 3-5 mice (mean \pm SEM). 
(G) $t$-SNE plot of graph-based clustering of myeloid cells sorted from peripheral blood of B16 tumor-bearing mice and processed for scRNA-seq (top), and expression levels of selected genes amongst myeloid clusters (bottom).

(H) Heatmap displaying expression levels of top 10 DE genes between blood myeloid cell clusters $(\mathbf{G})$ with genes ranked by fold change.

\section{Supplementary Figure 3}

(A) Heatmap displaying expression levels of selected "M1"- and "M2"-associated genes by Csf1r ${ }^{+}$Mafb $^{+}$cluster cells from B16 tumors (Fig 1C).

(B) $t$-SNE plot and graph-based clustering of myeloid cells sorted from PyMT tumors and processed for scRNA-seq (left) with expression levels of Csf1r (middle) and Mafb (right) displayed.

(C) Expression levels of B16 Csf1r Mafb $^{+}$cluster-specific gene signatures (Fig. 1E) displayed on $t$-SNE plots of $\mathrm{Csf1}^{+} \mathrm{Mafb}^{+}$clusters from PyMT tumors (Fig. 3C). Cells with top 70 percentile of signature expression level labeled in red.

(D) Heatmap displaying expression levels of B16 Csf1r ${ }^{+}$Mafb $^{+}$cluster-specific genes (Fig. 1E) by PyMT-infiltrating Csf1r ${ }^{+}$Mafb $^{+}$cells (Fig. $3 \mathrm{C}$ ).

(E) Expression of II7r (left) and Vcam1 (right) by cells in PyMT Csf1r ${ }^{+}$Mafb $^{+}$clusters (Fig. $3 C)$.

(F) Heatmap displaying expression levels of glycolysis- and oxidative phosphorylation (“OxPhos”)-associated genes in PyMT Csf1r ${ }^{+}$Mafb $^{+}$cluster cells (Fig. 3C).

\section{Supplementary Figure 4}


(A) Gating approach (left) and $t$-SNE plot (right) generated from flow cytometry analysis of the human RCC myeloid compartment. Example representative of more than 5 experiments.

(B) Expression levels of previously established blood myeloid cell signatures ${ }^{59}$ displayed on t-SNE plot of myeloid cells from human RCC biopsy (Fig. 4B).

(C) Expression levels of mouse B16 tumor myeloid cell signatures (Fig. 1E) displayed on tSNE plot of CSF1R MAFB $^{+}$clusters from human RCC biopsy (Fig. 4C).

(D) Heatmap of top 20 DE genes between human RCC stress-responsive (Cluster 4) and C1Q ${ }^{+}$TAM (Cluster 3) clusters (Fig. 4B). Genes ranked by fold change.

(E) Expression levels of "M1" (left) and "M2" (right) gene signatures (Fig. 3) displayed on $t$ SNE plot of CSF1R MAFB $^{+}$clusters from human RCC biopsy (Fig. 4C).

\section{Supplementary Figure 5}

(A) Quantification by flow cytometry of myeloid cell frequency within CD $45^{+}$cells from human RCC biopsies.

(B) Quantification of $T_{\text {reg }}$ cell number per milligram of B16 tumor from wildtype mice that were treated with anti-CTLA-4 antibodies (IgG2c or lgG1 clone) or corresponding isotype antibody controls (mean \pm SEM). Data was pooled from two independent experiments.

(C) Quantification of the ratio between macrophages (Ly6C- $\mathrm{F} 4 / 80^{+} \mathrm{CD}^{+} 4^{+}$) and monocytes $\left(\mathrm{Ly}_{6 \mathrm{C}} \mathrm{CD}^{+} \mathrm{Cb} \mathrm{b}^{+}\right)$in B16 tumors from wild-type mice treated with non-depleting antiCTLA-4 (IgG1 clone) or isotype antibody control. Data is representative of two independent experiments. ${ }^{* *} p<0.01,{ }^{* * *} p<0.001$.

(D) $t$-SNE plot of graph-based clustering of (left) and expression levels of select genes (right) of B16 tumor myeloid cells sorted from DT-treated FoxP3-DTR mice. 
(E) Heatmap displaying expression of top $10 \mathrm{DE}$ genes between B16-infiltrating Csf1r ${ }^{+}$ Mafb $^{+}$clusters aggregated from wildtype (Fig. 1C), DT-treated control and DT-treated FoxP3-DTR mice. Genes are ranked by fold change.

(F) Volcano plot displaying DE genes between B16 tumor $L y 6 c 2^{+} \mathrm{Hp}^{+}$monocytes from DTtreated control and FoxP3-DTR mice. Genes with $>0.4$ log-fold changes and an adjusted $\mathrm{p}$ value of 0.05 (based on Bonferroni correction), are highlighted in red. Genes of interest labeled.

\section{Supplementary Figure 6}

(A) Survival curves of kidney tumor patients whose TCGA tumor samples exhibited differential levels of the ratio of macrophage (Fig. 4E) to $\mathrm{CDC} 1^{53}$ gene signature scores. A score threshold of $50 \%$ (left), 33\% (middle), and 10\% (right) was used to determine high and low comparison groups.

(B) Survival curves of kidney tumor patients whose TCGA tumor samples exhibited differential levels of the cDC1 gene signature expression score ${ }^{53}$. A score threshold of $10 \%$ was used for patient group selection.

(C) Summary of hazard ratios and p-values from analyses of kidney tumor samples from $\operatorname{TCGA}(\mathbf{A}, \mathbf{B})$.

Table S1. DE genes between B16 tumor myeloid populations. DE analysis was performed between B16 tumor clusters (Fig. 1B). DE genes with $\log N$ fold change $>0.5$ and min.pct of 0.25 are listed. 
Table S2. DE genes between human RCC myeloid populations. DE analysis was performed on RCC myeloid clusters (Fig. 4B), and DE genes with $\log N$ fold change $>0.4$ and min.pct of 0.25 are listed.

Table S3. DE genes between monocyte/macrophage populations in control and Foxp3-

DTR tumors. DE analysis was performed on aggregated myeloid cell clusters from control and Foxp3-DTR (Fig. 5D). DE genes with log $N$ fold change $>0.001$ and min.pct of 0.1 listed. DE analysis for each cell cluster is listed on the specified tab. 


\section{References}

1. Merad, M., Sathe, P., Helft, J., Miller, J. \& Mortha, A. The Dendritic Cell Lineage: Ontogeny and Function of Dendritic Cells and Their Subsets in the Steady State and the Inflamed Setting. Annu Rev Immunol 31, 563-604 (2013).

2. Wculek, S. K. et al. Dendritic cells in cancer immunology and immunotherapy. Nat Rev Immunol 20, 7-24 (2020).

3. Lavin, Y., Mortha, A., Rahman, A. \& Merad, M. Regulation of macrophage development and function in peripheral tissues. Nat Rev Immunol 15, 731744 (2015).

4. Noy, R. \& Pollard, J. W. Tumor-associated macrophages: from mechanisms to therapy. Immunity 41, 4961 (2014).

5. DeNardo, D. G. \& Ruffell, B. Macrophages as regulators of tumour immunity and immunotherapy. Nat Rev Immunol 19, 369-382 (2019).

6. Schulz, C. et al. A Lineage of Myeloid Cells Independent of Myb and Hematopoietic Stem Cells. Science 336, 86-90 (2012).

7. Hashimoto, D. et al. Tissue-Resident Macrophages Self-Maintain Locally throughout Adult Life with Minimal Contribution from Circulating Monocytes. Immunity 38, 792-804 (2013).

8. Ginhoux, F. et al. Fate Mapping Analysis Reveals That Adult Microglia Derive from Primitive Macrophages. Science 330, 841-845 (2010).

9. Ginhoux, F. \& Guilliams, M. Tissue-Resident Macrophage Ontogeny and Homeostasis. Immunity 44, 439-449 (2016). 
10. Hoeffel, G. et al. C-Myb+ Erythro-Myeloid Progenitor-Derived Fetal Monocytes Give Rise to Adult Tissue-Resident Macrophages. Immunity 42, 665-678 (2015).

11. Franklin, R. A. et al. The cellular and molecular origin of tumor-associated macrophages.

Science 344, 921925 (2014).

12. Arwert, E. N. et al. A Unidirectional Transition from Migratory to Perivascular Macrophage Is Required for Tumor Cell Intravasation. Cell Reports 23, 1239-1248 (2018).

13. Qian, B.-Z. et al. CCL2 recruits inflammatory monocytes to facilitate breast-tumour metastasis. Nature 475, 222-225 (2011).

14. Loyher, P.-L. et al. Macrophages of distinct origins contribute to tumor development in the lung. J Exp Med 215, 2536-2553 (2018).

15. Casanova-Acebes, M. et al. Tissue-resident macrophages provide a pro-tumorigenic niche to early NSCLC cells. Nature 1-7 (2021) doi:10.1038/s41586-021-03651-8.

16. Zhu, Y. et al. Tissue-Resident Macrophages in Pancreatic Ductal Adenocarcinoma Originate from Embryonic Hematopoiesis and Promote Tumor Progression. Immunity 47, 323 338.e6 (2017).

17. Etzerodt, A. et al. Specific targeting of CD163+ TAMs mobilizes inflammatory monocytes and promotes T cell-mediated tumor regression. J Exp Med 216, 2394-2411 (2019).

18. Cheng, S. et al. A pan-cancer single-cell transcriptional atlas of tumor infiltrating myeloid cells. Cell 184, 792-809.e23 (2021). 
19. Gubin, M. M. et al. High-Dimensional Analysis Delineates Myeloid and Lymphoid Compartment Remodeling during Successful Immune-Checkpoint Cancer Therapy. Cell 175, 1014-1030.e19 (2018).

20. Zhang, L. et al. Single-Cell Analyses Inform Mechanisms of Myeloid-Targeted Therapies in Colon Cancer. Cell 181, 442-459.e29 (2020).

21. Guerriero, J. L. et al. Class Ila HDAC inhibition reduces breast tumours and metastases through anti-tumour macrophages. Nature 543, 428432 (2017).

22. Kaneda, M. M. et al. PI3Ky is a molecular switch that controls immune suppression. Nature 539, 437-442 (2016).

23. Henau, O. D. et al. Overcoming resistance to checkpoint blockade therapy by targeting PI3Ky in myeloid cells. Nature 539, 443-447 (2016).

24. Gordon, S. R. et al. PD-1 expression by tumour-associated macrophages inhibits phagocytosis and tumour immunity. Nature 545, 495499 (2017).

25. Baer, C. et al. Suppression of microRNA activity amplifies IFN- $\gamma$-induced macrophage activation and promotes anti-tumour immunity. Nat Cell Biol 18, 790-802 (2016).

26. Molgora, M. et al. TREM2 Modulation Remodels the Tumor Myeloid Landscape Enhancing Anti-PD-1 Immunotherapy. Cell 182, 886-900.e17 (2020).

27. Katzenelenbogen, Y. et al. Coupled scRNA-Seq and Intracellular Protein Activity Reveal an Immunosuppressive Role of TREM2 in Cancer. Cell 182, 872-885.e19 (2020).

28. Jakubzick, C. V., Randolph, G. J. \& Henson, P. M. Monocyte differentiation and antigenpresenting functions. Nat Rev Immunol 17, 349362 (2017). 
29. lijima, N., Mattei, L. M. \& Iwasaki, A. Recruited inflammatory monocytes stimulate antiviral Th1 immunity in infected tissue. Proc National Acad Sci 108, 284-289 (2011).

30. Plantinga, M. et al. Conventional and Monocyte-Derived CD11b+ Dendritic Cells Initiate and Maintain T Helper 2 Cell-Mediated Immunity to House Dust Mite Allergen. Immunity 38, 322335 (2013).

31. Hohl, T. M. et al. Inflammatory Monocytes Facilitate Adaptive CD4 T Cell Responses during Respiratory Fungal Infection. Cell Host Microbe 6, 470-481 (2009).

32. Theurl, I. et al. On-demand erythrocyte disposal and iron recycling requires transient macrophages in the liver. Nat Med 22, 945-951 (2016).

33. Nathan, C. F., Murray, H. W., Wiebe, M. E. \& Rubin, B. Y. Identification of interferon-gamma as the lymphokine that activates human macrophage oxidative metabolism and antimicrobial activity. J Exp Med 158, 670-689 (1983).

34. Stein, M., Keshav, S., Harris, N. \& Gordon, S. Interleukin 4 potently enhances murine macrophage mannose receptor activity: a marker of alternative immunologic macrophage activation. J Exp Med 176, 287-292 (1992).

35. Mills, C. D., Kincaid, K., Alt, J. M., Heilman, M. J. \& Hill, A. M. M-1/M-2 Macrophages and the Th1/Th2 Paradigm. J Immunol 164, 6166-6173 (2000).

36. Murray, P. J. et al. Macrophage Activation and Polarization: Nomenclature and Experimental Guidelines. Immunity 41, 14-20 (2014). 
37. Roberts, E. W. et al. Critical Role for CD103+/CD141+ Dendritic Cells Bearing CCR7 for Tumor Antigen Trafficking and Priming of T Cell Immunity in Melanoma. Cancer Cell 30, 324336 (2016).

38. Broz, M. L. et al. Dissecting the Tumor Myeloid Compartment Reveals Rare Activating Antigen-Presenting Cells Critical for T Cell Immunity. Cancer Cell 26, 638-652 (2014).

39. Binnewies, M. et al. Unleashing Type-2 Dendritic Cells to Drive Protective Antitumor CD4+ T Cell Immunity. Cell 177, 556-571.e16 (2019).

40. Salmon, H. et al. Expansion and Activation of CD103+ Dendritic Cell Progenitors at the Tumor Site Enhances Tumor Responses to Therapeutic PD-L1 and BRAF Inhibition. Immunity 44, 924-38 (2016).

41. Hildner, K. et al. Batf3 Deficiency Reveals a Critical Role for CD8a+ Dendritic Cells in Cytotoxic T Cell Immunity. Science 322, 1097-1100 (2008).

42. Ferris, S. T. et al. cDC1 prime and are licensed by CD4+ T cells to induce anti-tumour immunity. Nature 584, 624-629 (2020).

43. Maier, B. et al. A conserved dendritic-cell regulatory program limits antitumour immunity. Nature 580, 257-262 (2020).

44. Cassetta, L. \& Pollard, J. W. Targeting macrophages: therapeutic approaches in cancer. Nat Rev Drug Discov 17, 887-904 (2018).

45. Gabrilovich, D. I. \& Nagaraj, S. Myeloid-derived suppressor cells as regulators of the immune system. Nat Rev Immuno/ 9, 162-174 (2009). 
46. Ruffell, B. \& Coussens, L. M. Macrophages and therapeutic resistance in cancer. Cancer Cell 27, 462472 (2015).

47. Roxburgh, C. S. D. \& McMillan, D. C. The role of the in situ local inflammatory response in predicting recurrence and survival in patients with primary operable colorectal cancer. Cancer Treat Rev 38, 451-466 (2012).

48. Quatromoni, J. G. \& Eruslanov, E. Tumor-associated macrophages: function, phenotype, and link to prognosis in human lung cancer. Am J Transl Res 4, 376-89 (2012).

49. Edin, S. et al. The Distribution of Macrophages with a M1 or M2 Phenotype in Relation to Prognosis and the Molecular Characteristics of Colorectal Cancer. Plos One 7, e47045 (2012).

50. Krieg, C. et al. High-dimensional single-cell analysis predicts response to anti-PD-1 immunotherapy. Nat Med 24, 144-153 (2018).

51. Combes, A. J. et al. A Pan-Cancer Census of Dominant Tumor Immune Archetypes. Biorxiv 2021.04.26.441344 (2021) doi:10.1101/2021.04.26.441344.

52. Miller, J. C. et al. Deciphering the transcriptional network of the dendritic cell lineage. Nat Immunol 13, 888899 (2012).

53. Barry, K. C. et al. A natural killer-dendritic cell axis defines checkpoint therapy-responsive tumor microenvironments. Nat Med 24, 1178-1191 (2018).

54. Trapnell, C. et al. The dynamics and regulators of cell fate decisions are revealed by pseudotemporal ordering of single cells. Nat Biotechnol 32, 381-386 (2014).

55. Azizi, E. et al. Single-Cell Map of Diverse Immune Phenotypes in the Breast Tumor Microenvironment. Cell 174, 1293-1308.e36 (2018). 
56. O’Neill, L. A. J., Kishton, R. J. \& Rathmell, J. A guide to immunometabolism for immunologists. Nat Rev Immunol 16, 553-565 (2016).

57. Argüello, R. J. et al. SCENITH: A Flow Cytometry-Based Method to Functionally Profile Energy Metabolism with Single-Cell Resolution. Cell Metab 32, 1063-1075.e7 (2020).

58. Chevrier, S. et al. An Immune Atlas of Clear Cell Renal Cell Carcinoma. Cell 169, 736749.e18 (2017).

59. Villani, A.-C. et al. Single-cell RNA-seq reveals new types of human blood dendritic cells, monocytes, and progenitors. Science 356, eaah4573 (2017).

60. Braun, D. A. et al. Progressive immune dysfunction with advancing disease stage in renal cell carcinoma. Cancer Cell (2021) doi:10.1016/j.ccell.2021.02.013.

61. Roberts, A. W. et al. Tissue-Resident Macrophages Are Locally Programmed for Silent Clearance of Apoptotic Cells. Immunity 47, 913-927.e6 (2017).

62. Barrett, C. W. et al. Selenoprotein P influences colitis-induced tumorigenesis by mediating stemness and oxidative damage. J Clin Invest 125, 2646-2660 (2015).

63. Bos, P. D., Plitas, G., Rudra, D., Lee, S. Y. \& Rudensky, A. Y. Transient regulatory T cell ablation deters oncogene-driven breast cancer and enhances radiotherapy. J Exp Med 210, 2435-2466 (2013).

64. Newman, A. M. et al. Robust enumeration of cell subsets from tissue expression profiles. Nat Methods 12, 453-457 (2015).

65. Krummel, M. F. \& Allison, J. P. CD28 and CTLA-4 have opposing effects on the response of T cells to stimulation. J Exp Medicine 182, 459465 (1995). 
66. Furth, R. van \& Cohn, Z. A. The Origin and Kinetics of Mononuclear Phagocytes. J Exp Medicine 128, 415-435 (1968).

67. Jakubzick, C. et al. Minimal Differentiation of Classical Monocytes as They Survey SteadyState Tissues and Transport Antigen to Lymph Nodes. Immunity 39, 599-610 (2013).

68. Sen, D. et al. Tracking the Spatial and Functional Gradient of Monocyte-To-Macrophage Differentiation in Inflamed Lung. Plos One 11, e0165064 (2016).

69. $\mathrm{Bi}, \mathrm{K}$. et al. Tumor and immune reprogramming during immunotherapy in advanced renal cell carcinoma. Cancer Cell (2021) doi:10.1016/j.ccell.2021.02.015.

70. Kiner, E. et al. Gut CD4+ T cell phenotypes are a continuum molded by microbes, not by TH archetypes. Nat Immunol 22, 216-228 (2021).

71. Pandey, A. et al. Cloning of a receptor subunit required for signaling by thymic stromal lymphopoietin. Nat Immunol 1, 59-64 (2000).

72. Park, L. S. et al. Cloning of the Murine Thymic Stromal Lymphopoietin (Tslp) Receptor. J Exp Medicine 192, 659-670 (2000).

73. Corcoran, S. E. \& O’Neill, L. A. J. HIF1 $\alpha$ and metabolic reprogramming in inflammation. J Clin Invest 126, 3699-3707 (2016).

74. Keren, L. et al. A Structured Tumor-Immune Microenvironment in Triple Negative Breast Cancer Revealed by Multiplexed Ion Beam Imaging. Cell 174, 1373-1387.e19 (2018).

75. Angelo, M. et al. Multiplexed ion beam imaging of human breast tumors. Nat Med 20, 436442 (2014). 
76. Asp, M. et al. A Spatiotemporal Organ-Wide Gene Expression and Cell Atlas of the Developing Human Heart. Cell 179, 1647-1660.e19 (2019).

77. Hu, K. H. et al. ZipSeq: barcoding for real-time mapping of single cell transcriptomes. Nat Methods 17, 833-843 (2020).

78. Lee, Y. et al. XYZeq: Spatially resolved single-cell RNA sequencing reveals expression heterogeneity in the tumor microenvironment. Sci Adv 7, eabg4755 (2021).

79. Kim, J. M., Rasmussen, J. P. \& Rudensky, A. Y. Regulatory T cells prevent catastrophic autoimmunity throughout the lifespan of mice. Nat Immunol 8, 191-197 (2007).

80. Plitas, G. \& Rudensky, A. Y. Regulatory T Cells in Cancer. Annu. Rev. Cancer Biol. 4, 459477 (2020).

81. Vignali, D. A. A., Collison, L. W. \& Workman, C. J. How regulatory T cells work. Nat Rev Immunol 8, 523-532 (2008).

82. Liu, C. et al. Treg Cells Promote the SREBP1-Dependent Metabolic Fitness of TumorPromoting Macrophages via Repression of CD8+ T Cell-Derived Interferon-y. Immunity 51, 381397.e6 (2019).

83. Cipolletta, D., Cohen, P., Spiegelman, B. M., Benoist, C. \& Mathis, D. Appearance and disappearance of the mRNA signature characteristic of Treg cells in visceral adipose tissue: age, diet, and PPARy effects. Proc National Acad Sci 112, 482487 (2015).

84. Lumeng, C. N., Bodzin, J. L. \& Saltiel, A. R. Obesity induces a phenotypic switch in adipose tissue macrophage polarization. J Clin Invest 117, 175-184 (2007). 
85. Burzyn, D. et al. A special population of regulatory T cells potentiates muscle repair. Cell 155, 12821295 (2013).

86. Yan, X. et al. Temporal dynamics of cardiac immune cell accumulation following acute myocardial infarction. J Mol Cell Cardiol 62, 24-35 (2013).

87. Weirather, J. et al. Foxp3+ CD4+ T Cells Improve Healing After Myocardial Infarction by Modulating Monocyte/Macrophage Differentiation. Circ Res 115, 55-67 (2014).

88. Panduro, M., Benoist, C. \& Mathis, D. Treg cells limit IFN-y production to control macrophage accrual and phenotype during skeletal muscle regeneration. Proc National Acad Sci 115, 201800618 (2018).

89. Mujal, A. M. \& Krummel, M. F. Immunity as a continuum of archetypes. Science 364, 28-29 (2019).

90. DeNardo, D. G. et al. Leukocyte Complexity Predicts Breast Cancer Survival and Functionally Regulates Response to Chemotherapy. Cancer Discov 1, 54-67 (2011).

91. Mondini, M. et al. CCR2-dependent recruitment of Tregs and monocytes following radiotherapy is associated with TNFa-mediated resistance. Cancer Immunol Res 7, canimm.0633.2018 (2019).

92. Ruffell, B. et al. Macrophage IL-10 blocks CD8+ T cell-dependent responses to chemotherapy by suppressing IL-12 expression in intratumoral dendritic cells. Cancer Cell 26, 623637 (2014). 
93. Medrek, C., Pontén, F., Jirström, K. \& Leandersson, K. The presence of tumor associated macrophages in tumor stroma as a prognostic marker for breast cancer patients. Bmc Cancer 12, 306 (2012).

94. Krishna, C. et al. Single-cell sequencing links multiregional immune landscapes and tissueresident T cells in ccRCC to tumor topology and therapy efficacy. Cancer Cell 39, 662-677.e6 (2021).

95. Hakimi, A. A. et al. Transcriptomic Profiling of the Tumor Microenvironment Reveals Distinct Subgroups of Clear cell Renal Cell Cancer - Data from a Randomized Phase III Trial. Cancer Discov 9, CD-18-0957 (2019).

96. McDermott, D. F. et al. Clinical activity and molecular correlates of response to atezolizumab alone or in combination with bevacizumab versus sunitinib in renal cell carcinoma. Nat Med 24, 749-757 (2018).

97. Soudja, S. M., Ruiz, A. L., Marie, J. C. \& Lauvau, G. Inflammatory Monocytes Activate Memory CD8+ T and Innate NK Lymphocytes Independent of Cognate Antigen during Microbial Pathogen Invasion. Immunity 37, 549-562 (2012).

98. Leirião, P., Fresno, C. del \& Ardavín, C. Monocytes as effector cells: Activated Ly-6Chigh mouse monocytes migrate to the lymph nodes through the lymph and cross-present antigens to CD8+T cells. Eur J Immunol 42, 2042-2051 (2012).

99. Bain, C. C. et al. Resident and pro-inflammatory macrophages in the colon represent alternative context-dependent fates of the same Ly6Chi monocyte precursors. Mucosal Immunol 6, 498-510 (2013). 
100. Tamoutounour, S. et al. CD64 distinguishes macrophages from dendritic cells in the gut and reveals the Th1-inducing role of mesenteric lymph node macrophages during colitis. Eur $J$ Immunol 42, 3150-3166 (2012).

101. Engelhardt, J. J. et al. Marginating Dendritic Cells of the Tumor Microenvironment CrossPresent Tumor Antigens and Stably Engage Tumor-Specific T Cells. Cancer Cell 21, 402417 (2012).

102. Headley, M. B. et al. Visualization of immediate immune responses to pioneer metastatic cells in the lung. Nature 531, 513517 (2016).

103. Dobin, A. et al. STAR: ultrafast universal RNA-seq aligner. Bioinformatics 29, 15-21 (2013).

104. Satija, R., Farrell, J. A., Gennert, D., Schier, A. F. \& Regev, A. Spatial reconstruction of single-cell gene expression data. Nat Biotechnol 33, 495-502 (2015).

105. Kowalczyk, M. S. et al. Single-cell RNA-seq reveals changes in cell cycle and differentiation programs upon aging of hematopoietic stem cells. Genome Res 25, 18601872 (2015).

106. Macosko, E. Z. et al. Highly Parallel Genome-wide Expression Profiling of Individual Cells Using Nanoliter Droplets. Cell 161, 12021214 (2015).

107. Stuart, T. et al. Comprehensive Integration of Single-Cell Data. Cell 177, 1888-1902.e21 (2019).

108. Dominguez, D. et al. A high-resolution transcriptome map of cell cycle reveals novel connections between periodic genes and cancer. Cell Res 26, 946-962 (2016). 
109. Hafemeister, C. \& Satija, R. Normalization and variance stabilization of single-cell RNA-seq data using regularized negative binomial regression. Genome Biol 20, 296 (2019).

110. Qiu, X. et al. Reversed graph embedding resolves complex single-cell trajectories. Nat Methods 14, 979-982 (2017).

111. Vivian, J. et al. Toil enables reproducible, open source, big biomedical data analyses. Nat Biotechnol 35, 314-316 (2017).

112. Goldman, M. J. et al. Visualizing and interpreting cancer genomics data via the Xena platform. Nat Biotechnol 38, 675-678 (2020).

113. Robinson, M. D. \& Oshlack, A. A scaling normalization method for differential expression analysis of RNA-seq data. Genome Biol 11, R25 (2010).

114. Virtanen, P. et al. SciPy 1.0: fundamental algorithms for scientific computing in Python. Nat Methods 17, 261-272 (2020). 
A

FACS isolation

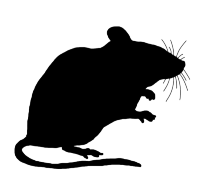

$\mathrm{C} 57 \mathrm{BL} / 6$

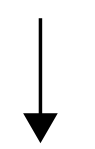

Harvest B16F10

s.c. tumors

C

for single-cell RNA-seq.

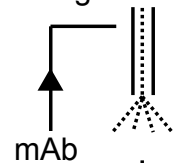

stain

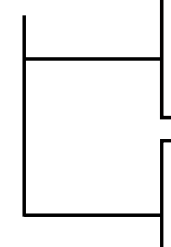

Ly6C
( Mono,

Ly6C- MHC-II+
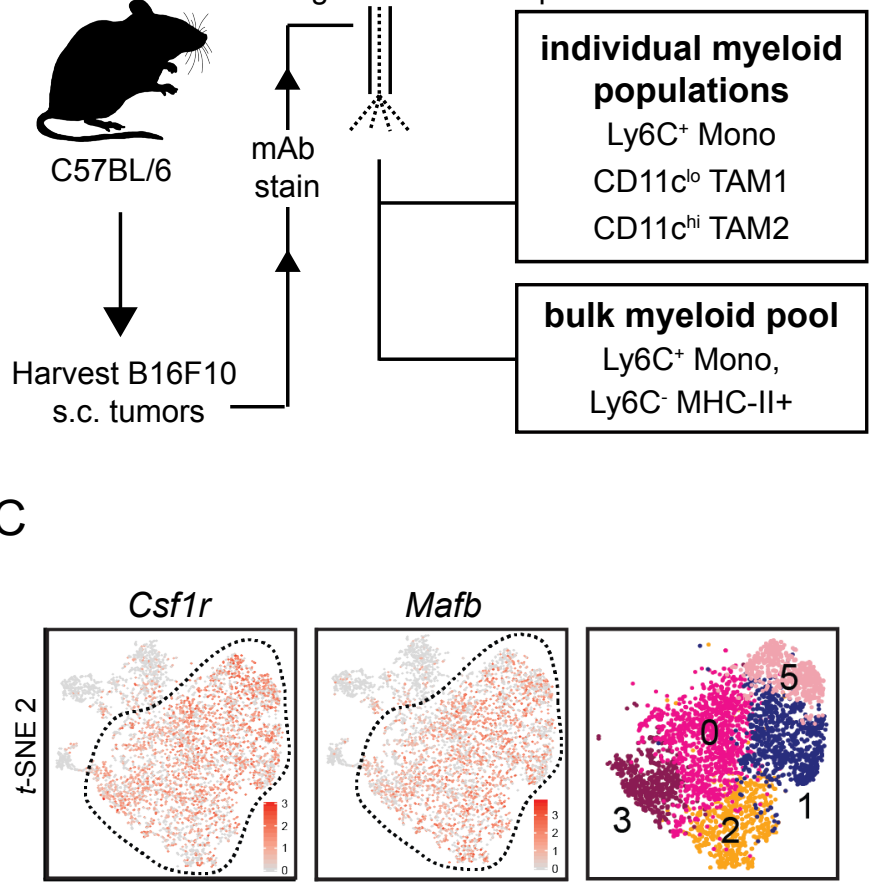

$t$-SNE 1

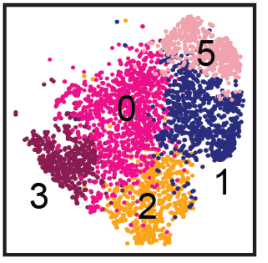

E

Bulk B16 Tumor:

Csf1r ${ }^{+} \mathrm{Mafb}^{+}$Clusters

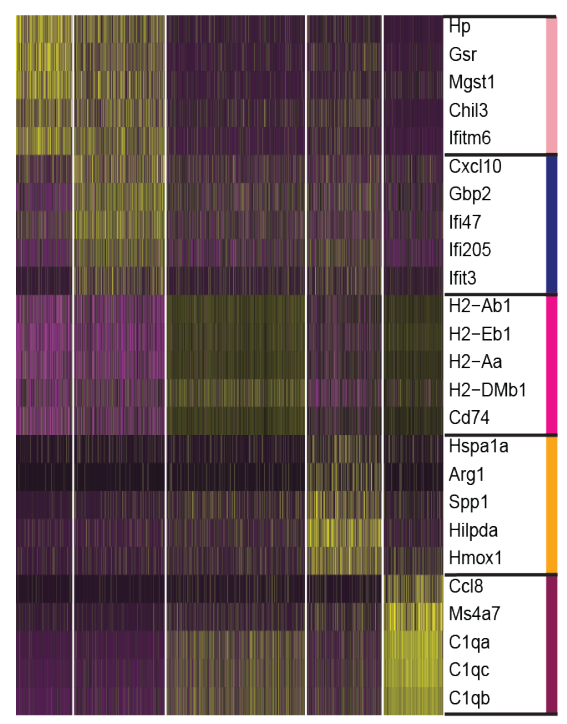

$\begin{array}{lllll}5 & 1 & 0 & 2 & 3\end{array}$

G

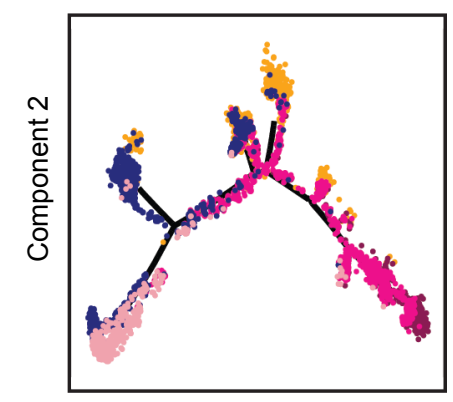

Component 1
$L y 6 c 2^{+} H p^{+}$

Mono

IFN-resp.

Mono

$L y 6 c 2^{+} H 2-A b 1^{+}$

Mono-Int

Stress-resp.

C1qa+

TAM

B

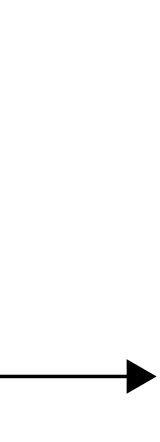

D

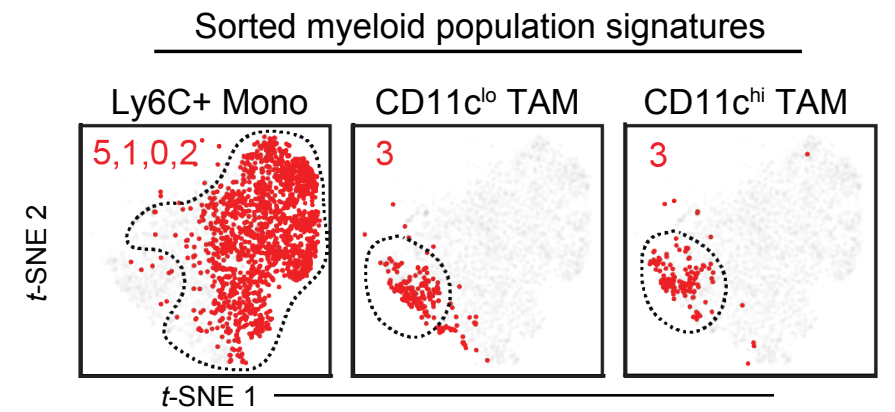

$\mathrm{F}$

Ly6c2

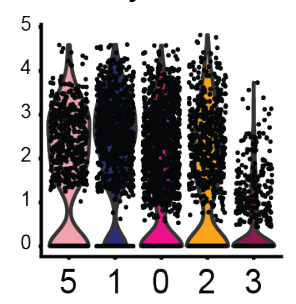

Cxcl10

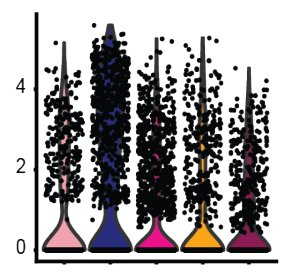

51023
Bulk B16 tumor:

Mono \& Ly6C- MHC-II+ cells

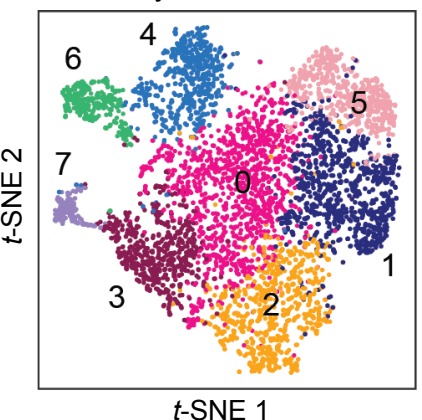

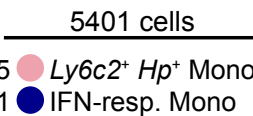

0 Mono-Int

2 Stress-resp

$3 . \mathrm{C}_{\mathrm{qqa}} \mathrm{T}^{\mathrm{TAM}}$

$4 \mathrm{cDC} 2$

$7 \bigcirc \mathrm{cDC} 1$

$6 \bigcirc \mathrm{Crr}^{+} \mathrm{cDC}$

$t$-SNE 1

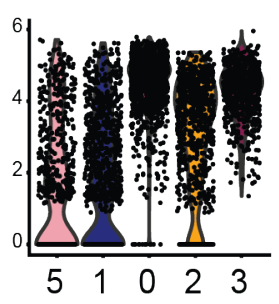

Arg1

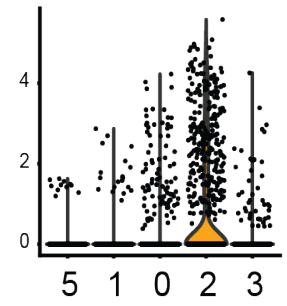

$H p$

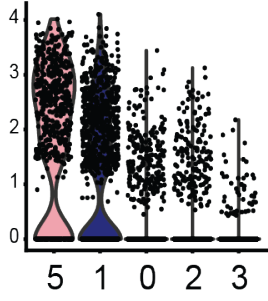

C1qa

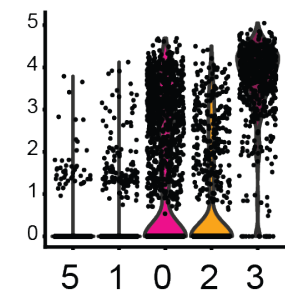

$H$

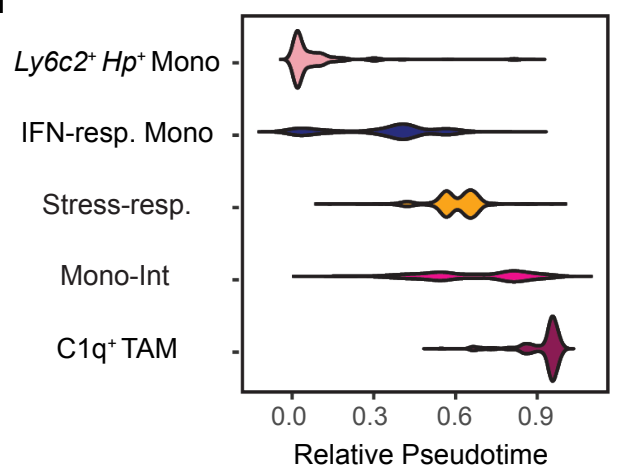

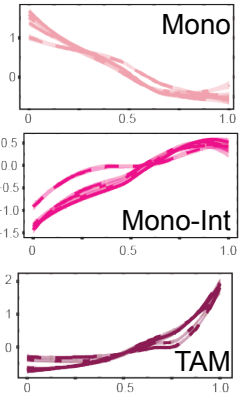

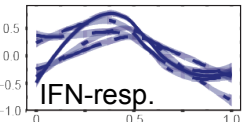

Relative Pseudotime 
bioRxiv preprint doi: https://doi.org/10.1101/2021.07.07.451502; this version posted July 8, 2021. The copyright holder for this preprint (which was not certified by peer review) is the author/funder. All rights reserved. No reuse allowed without permission.

A FACS-isolated Ly6C+ Mono

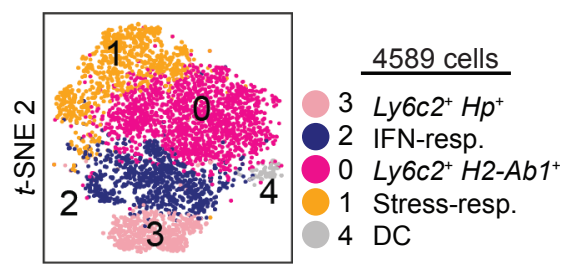

t-SNE 1

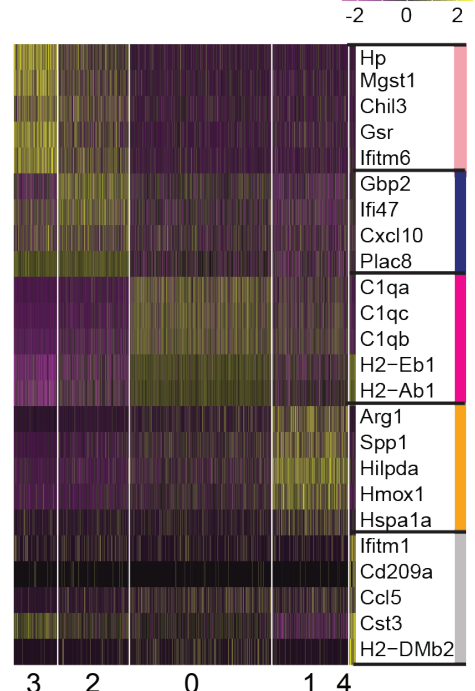

D

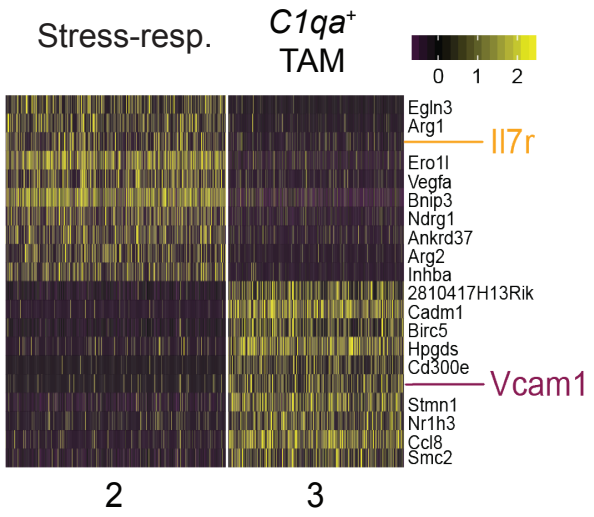

G
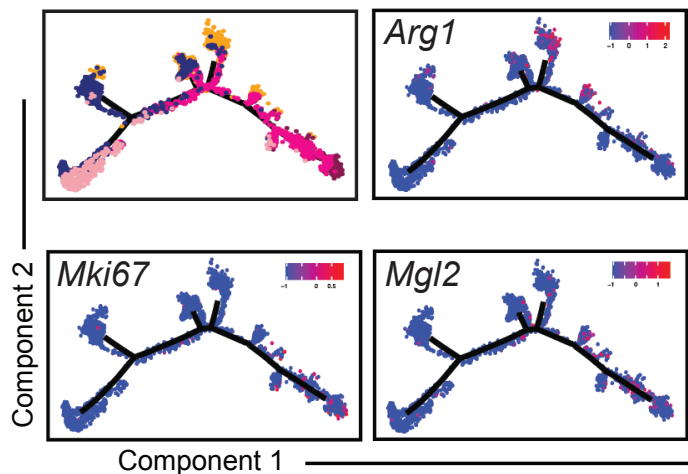

E

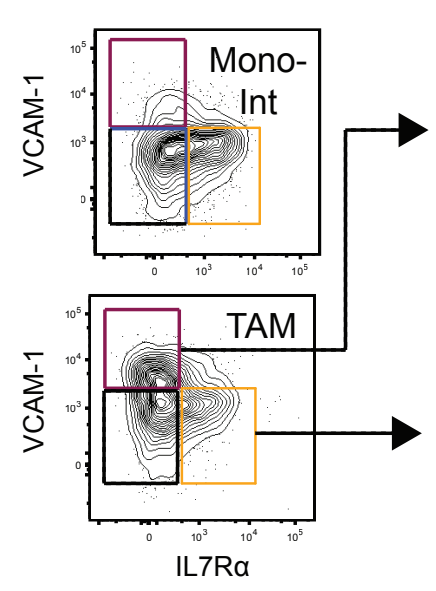

$\mathrm{H}$
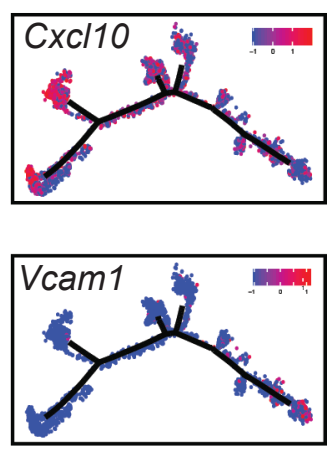

C FACS-isolated CD11 ${ }^{\text {hi }}$ TAM2

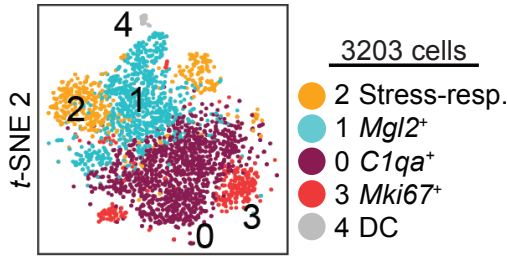

t-SNE 1
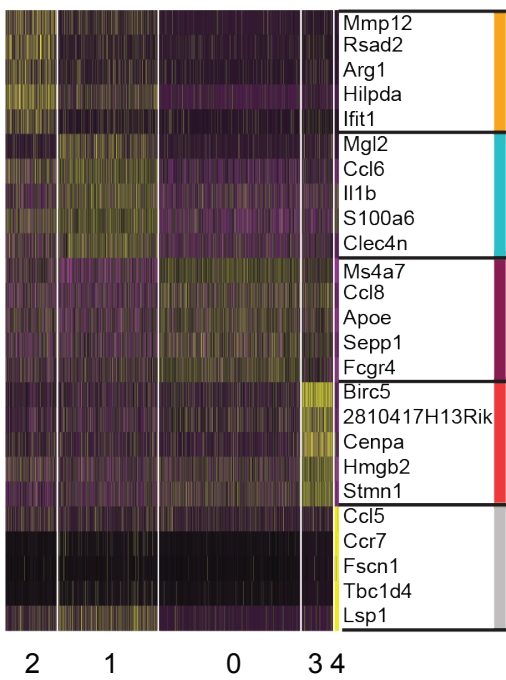

F

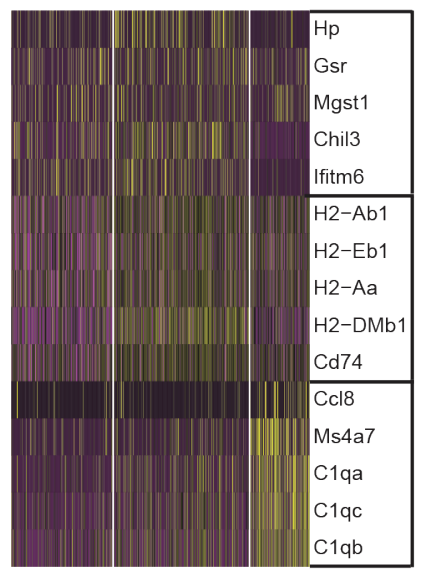

$\begin{array}{lll}2.1 & 2.0 & 2.2\end{array}$

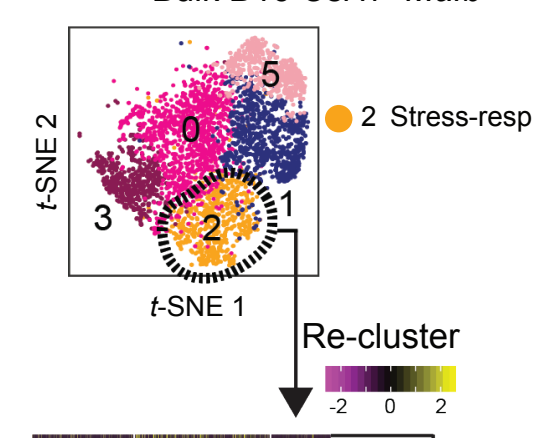

\section{Bulk B16 Csf1r Mafb $^{+}$}


A

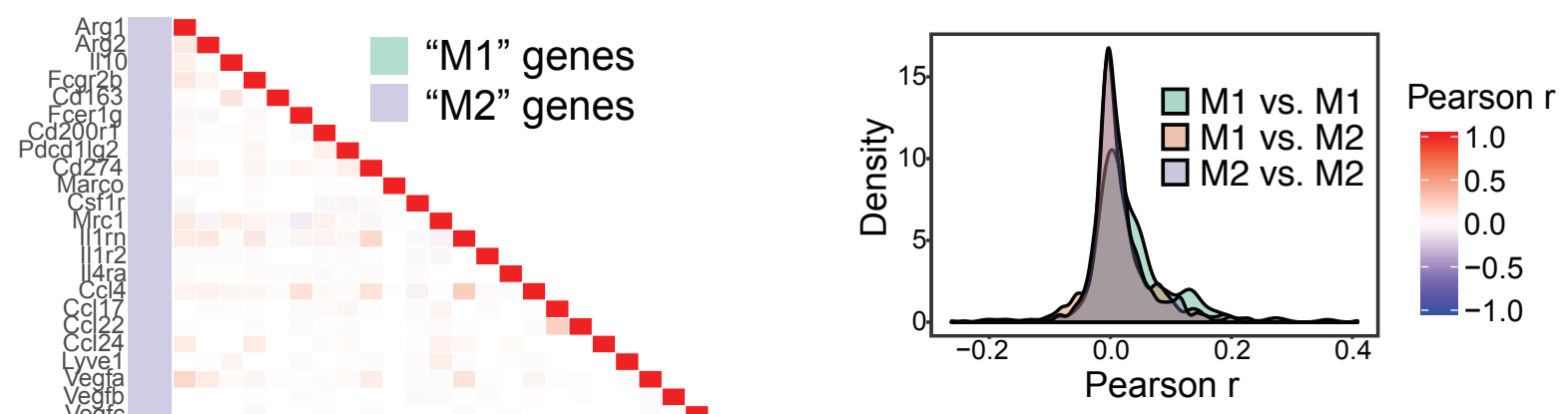

B

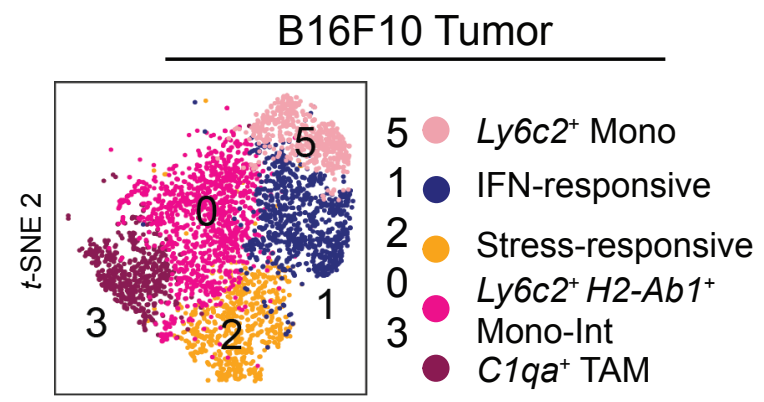

t-SNE 1

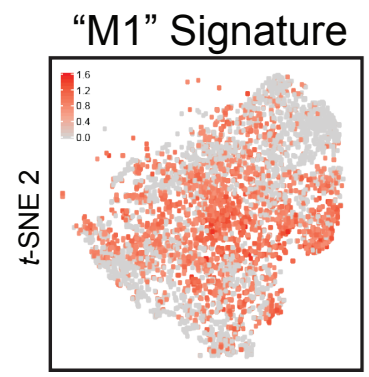

D

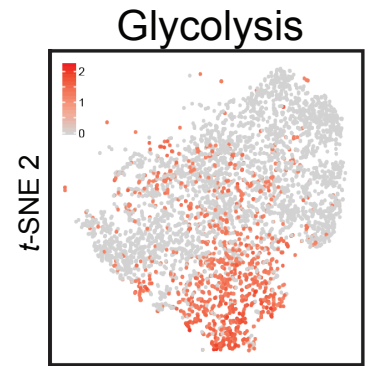

t-SNE 1

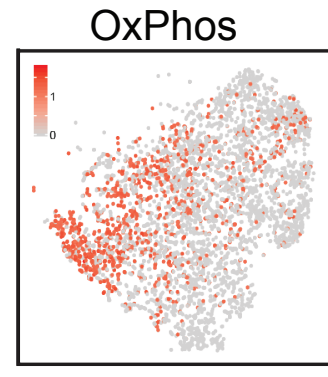

"M2" Signature

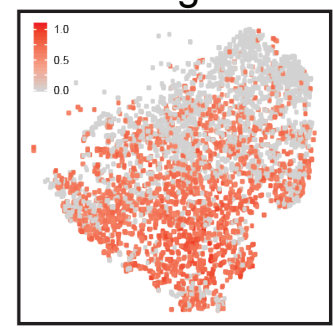

biti

t-SNE 1
C
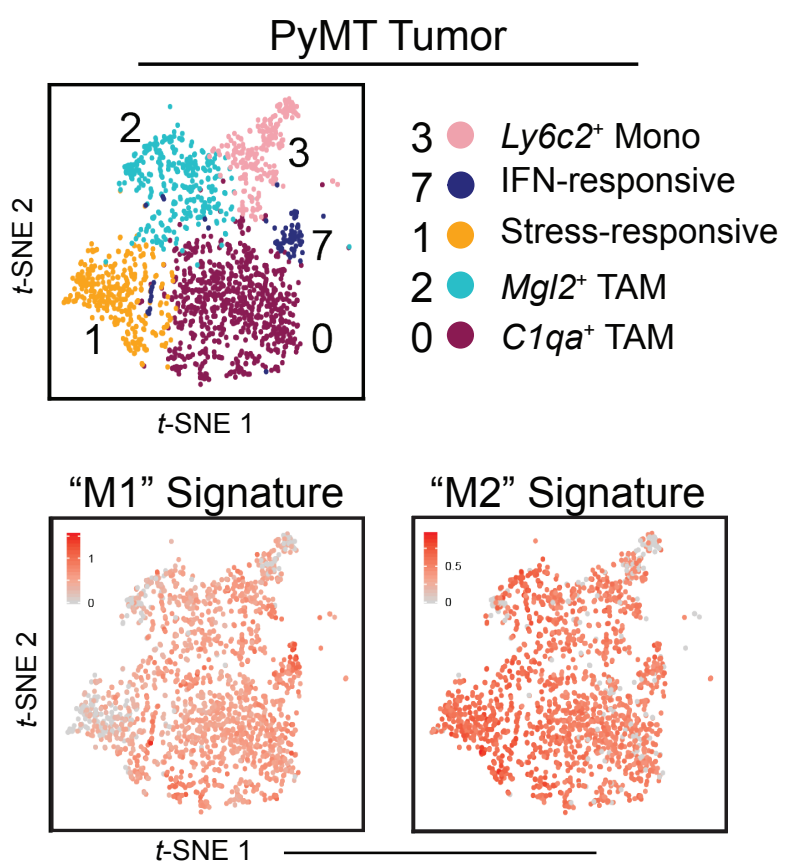

E

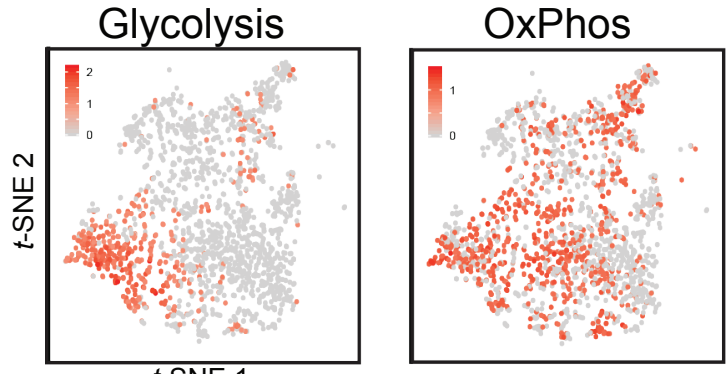


bioRxiv preprint doi: https://doi.org/10.1101/2021.07.07.451502; this version posted July 8, 2021. The copyright holder for this preprint (which was not certified by peer review) is the author/funder. All rights reserved. No reuse allowed without permission.

A

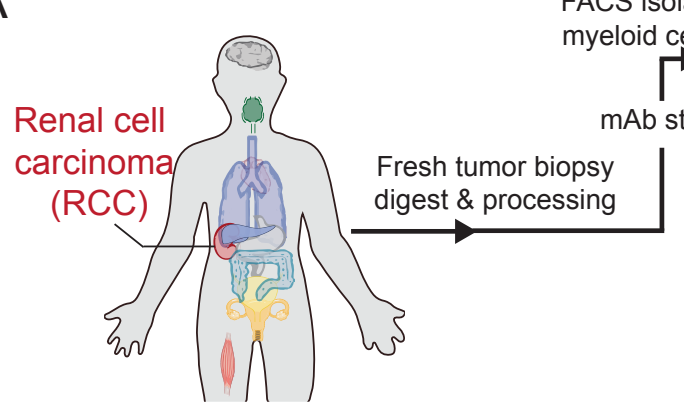

C

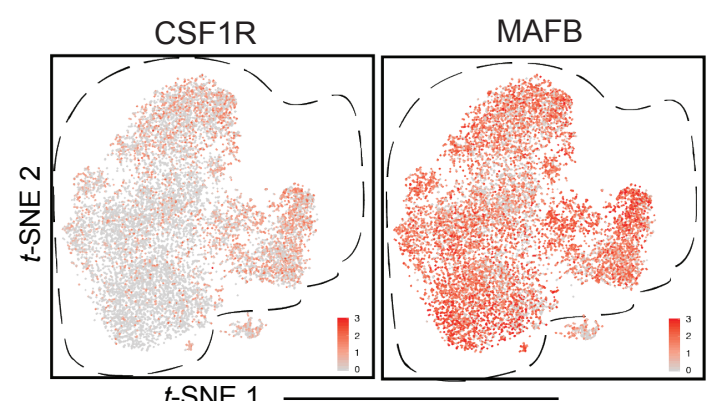

D

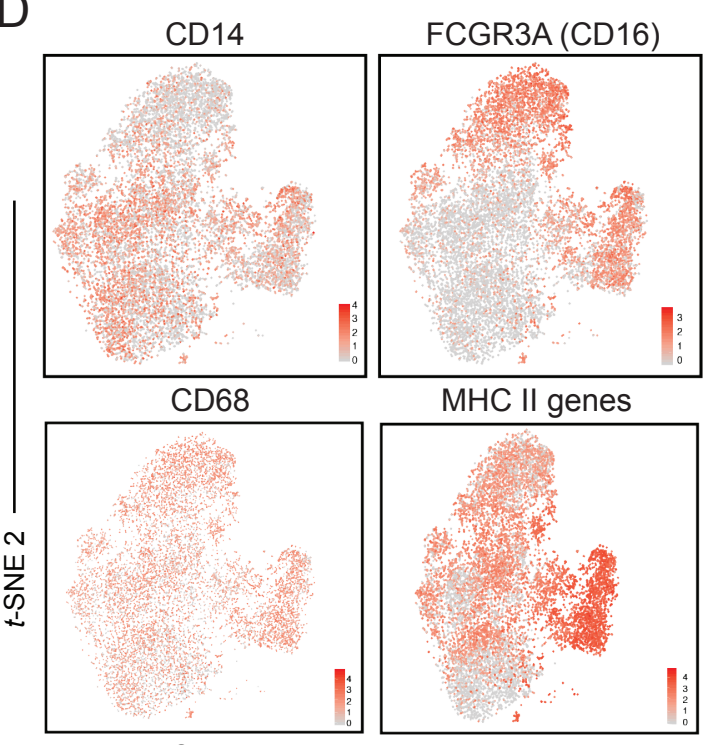

t-SNE 1

G

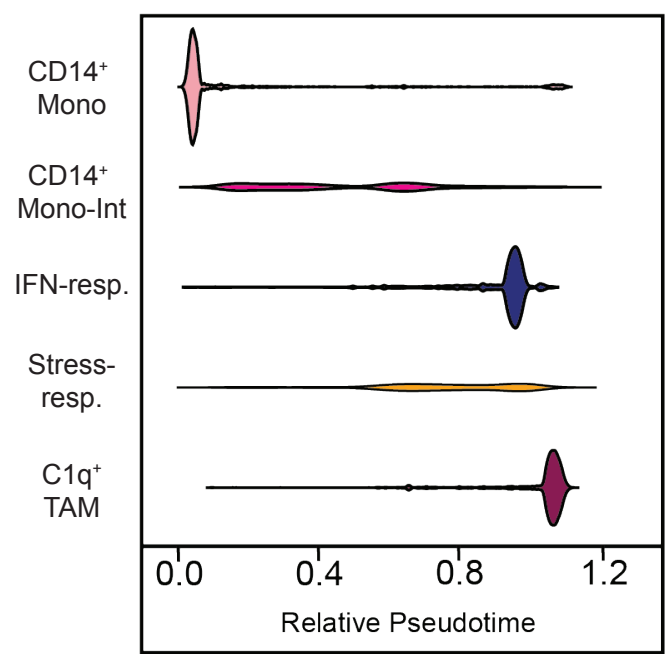

B

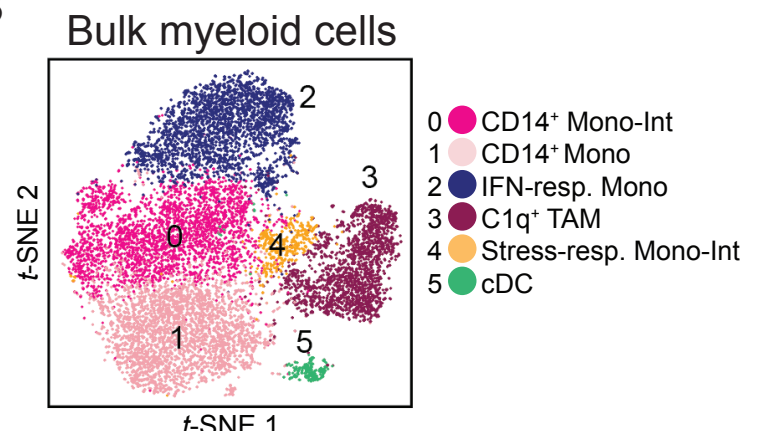

E
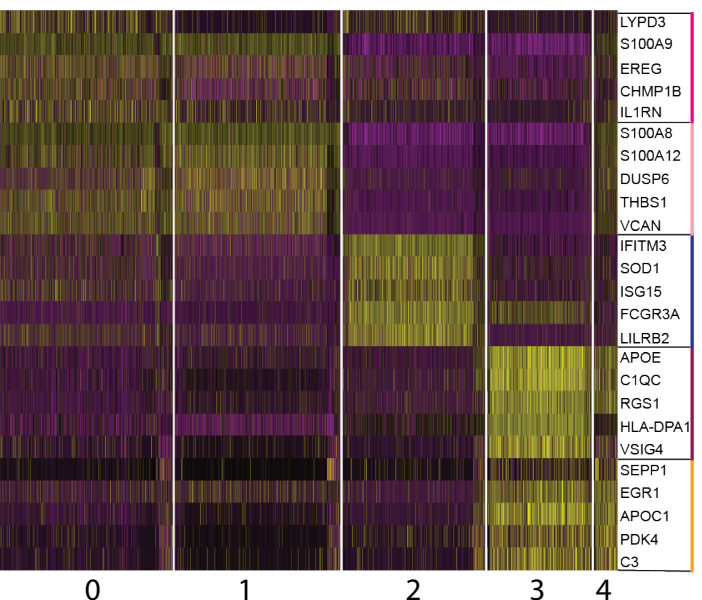

CD14

Mono-Int

CD14+

Monocytes

IFN-responsive Monocytes

$\mathrm{C} 1 \mathrm{q}^{+}$

TAM

Stress-responsive Mono-Int

F
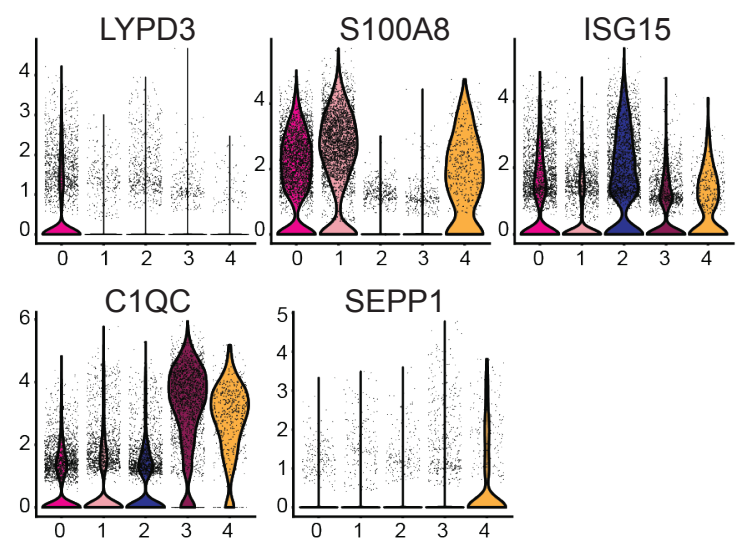

$\mathrm{H}$

Glycolysis Score

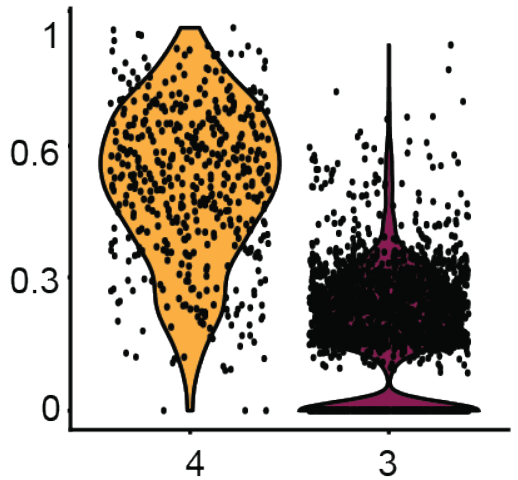

Mujal AM, Combes AJ, et al. 
A
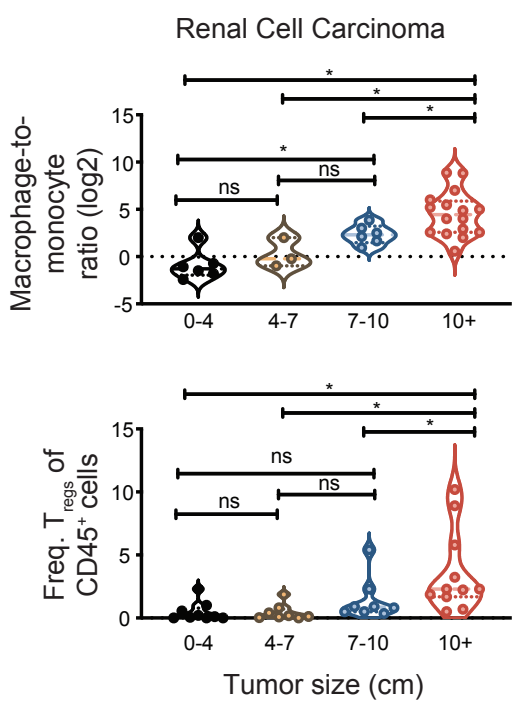

$\mathrm{D}$

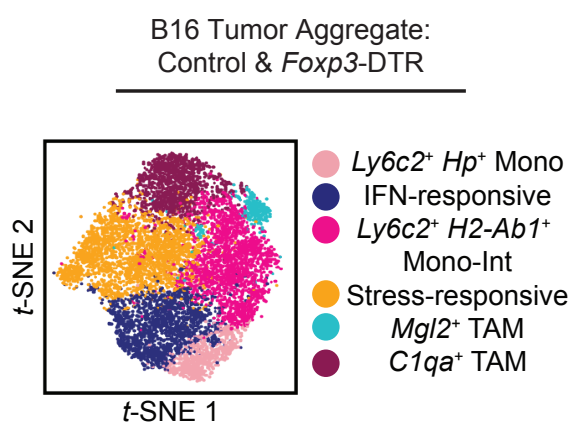

Control

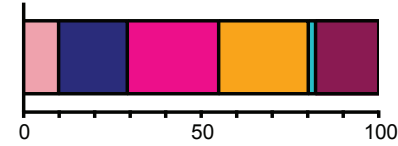

Foxp3-

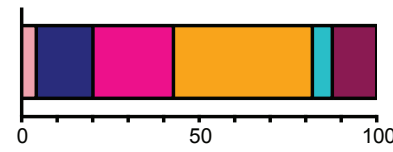

Freq. of cells

F
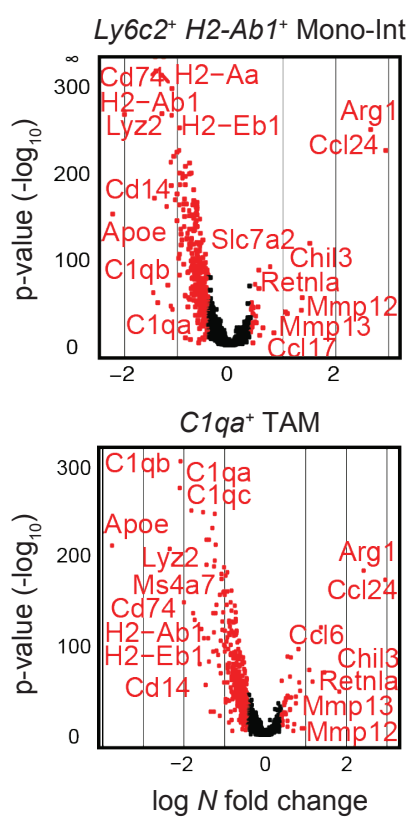

Mujal AM, Combes AJ, et al.

B

G

$\mathrm{H}$
Renal Cell Carcinoma

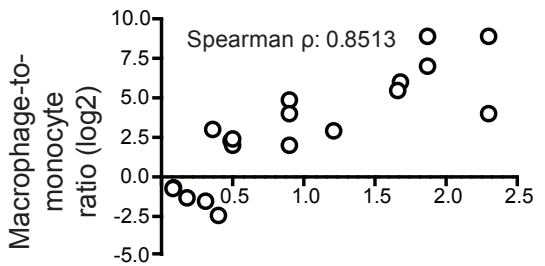

Melanoma

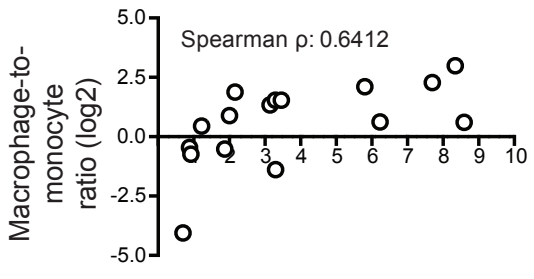

Freq. $T_{\text {regs }}$ of $C D 45^{+}$cells

E
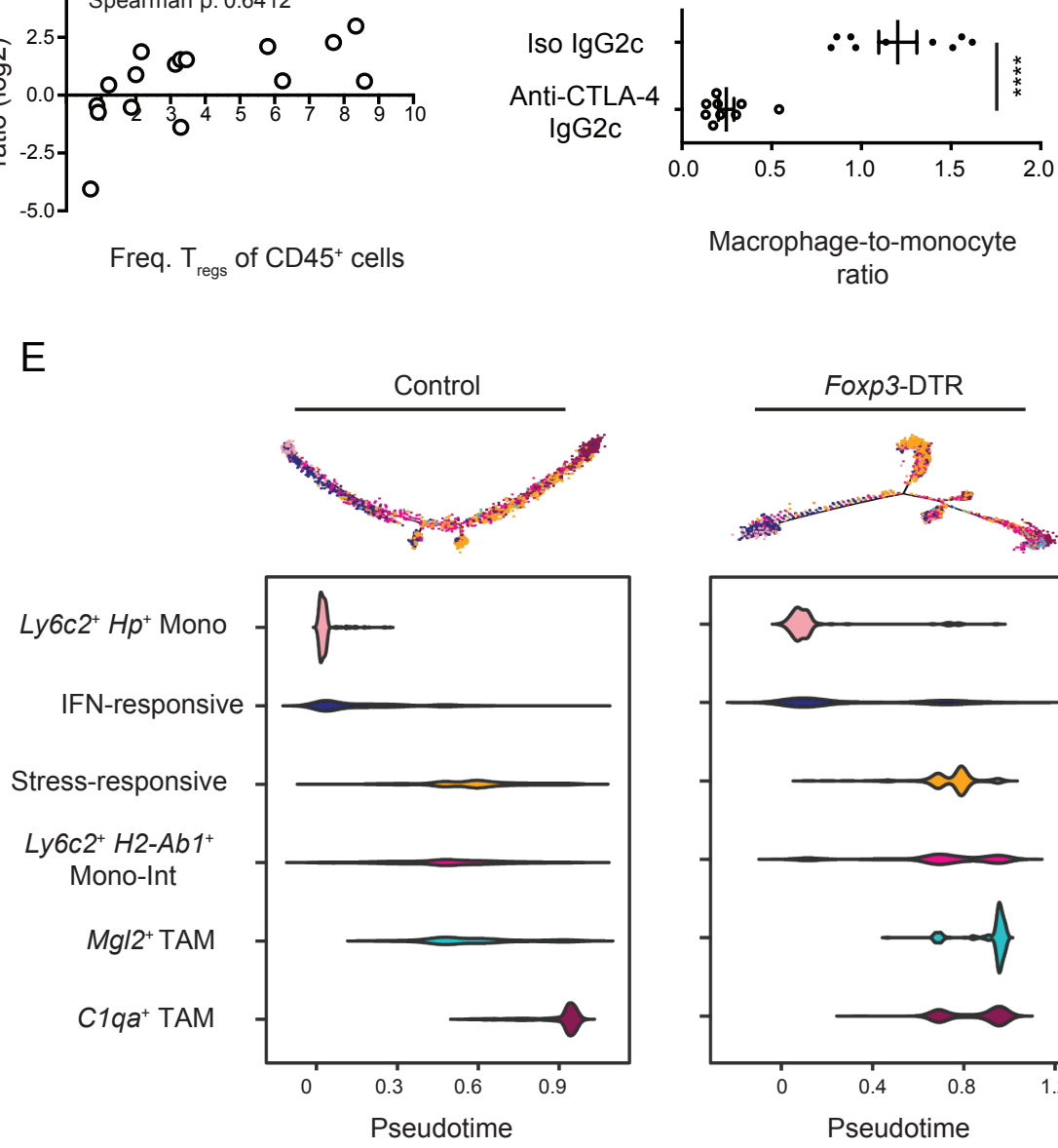

B16 Tumor

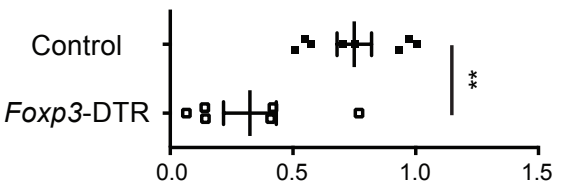

ratio
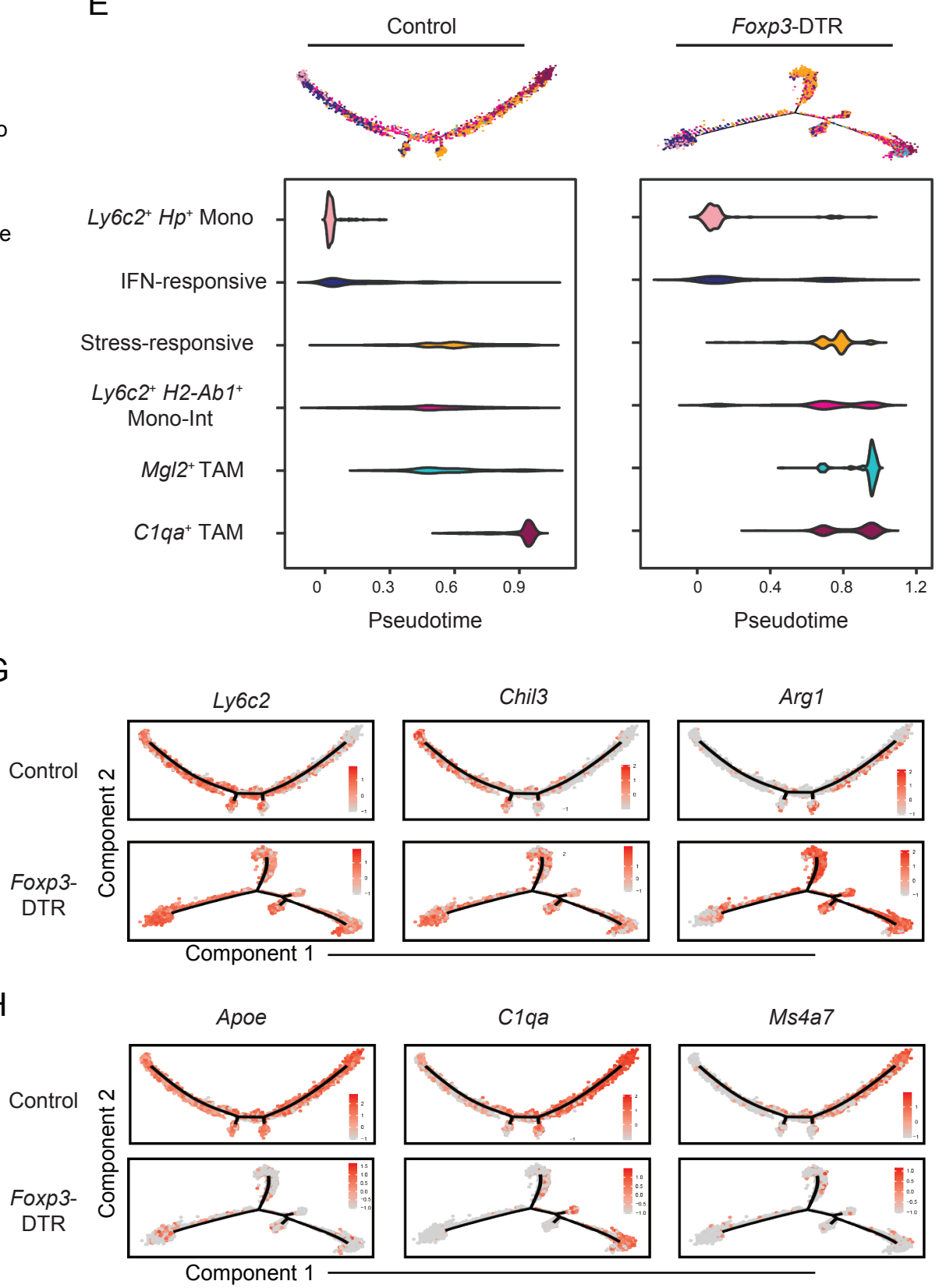

C1qa
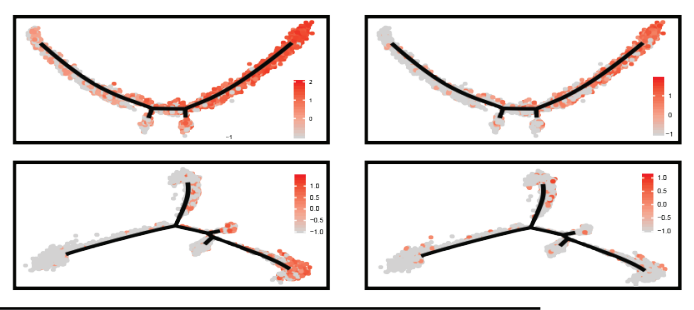

Figure 5 
A

\section{TCGA Overall Survival in Kidney Tumors}

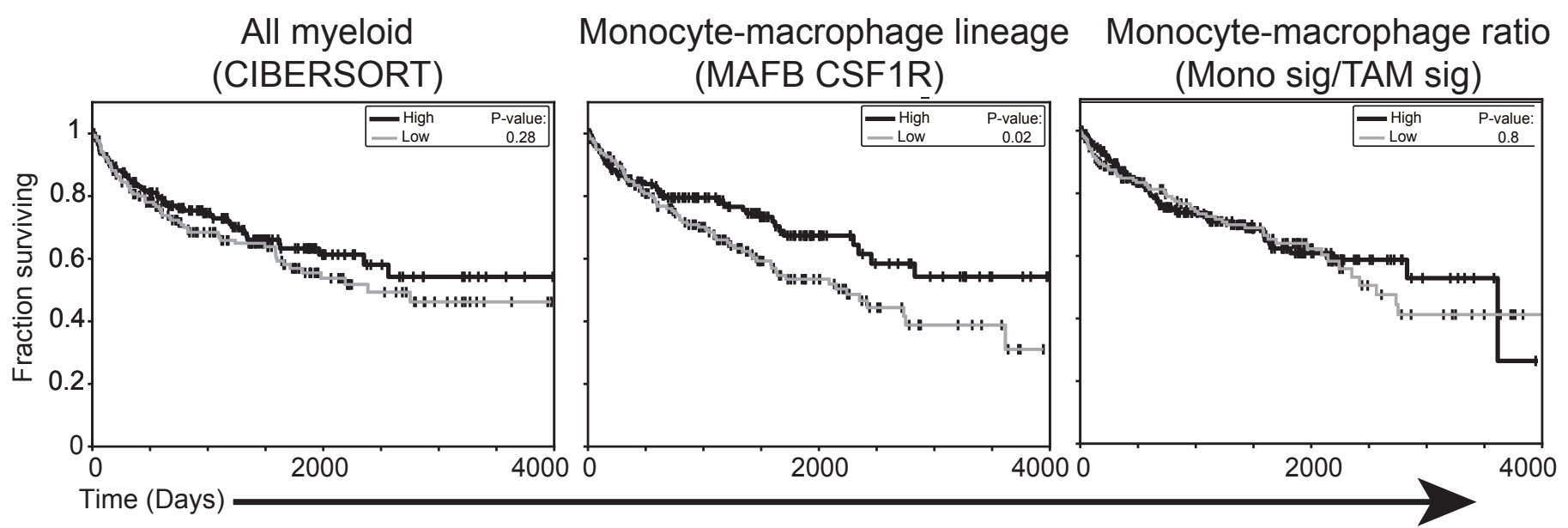

B

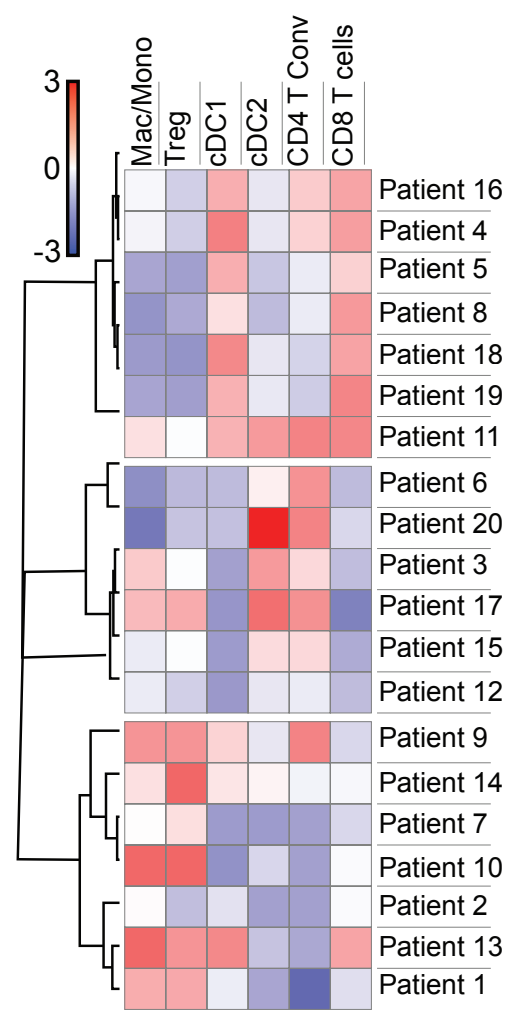

C

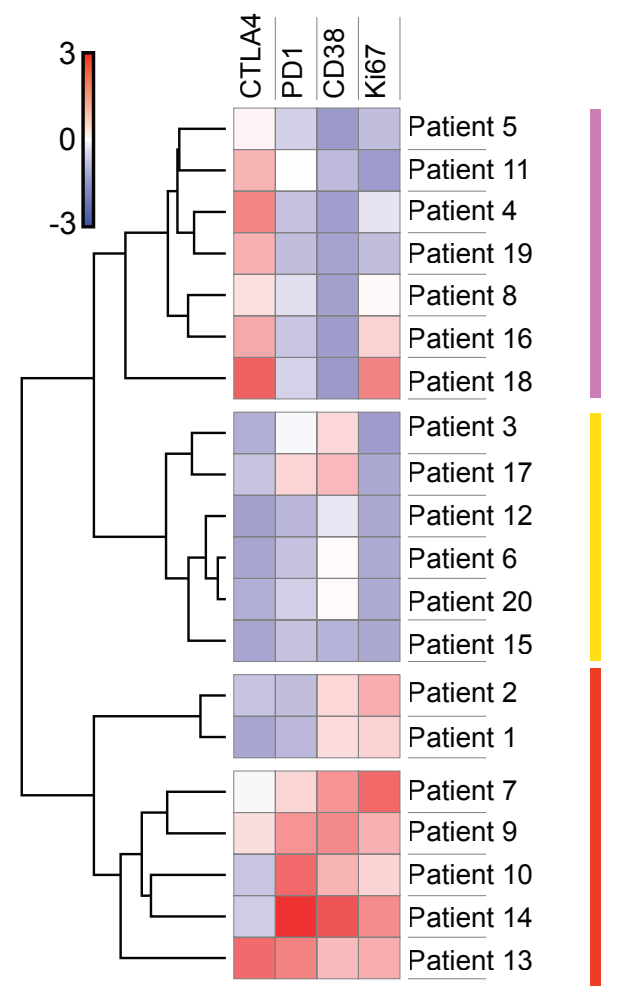

E

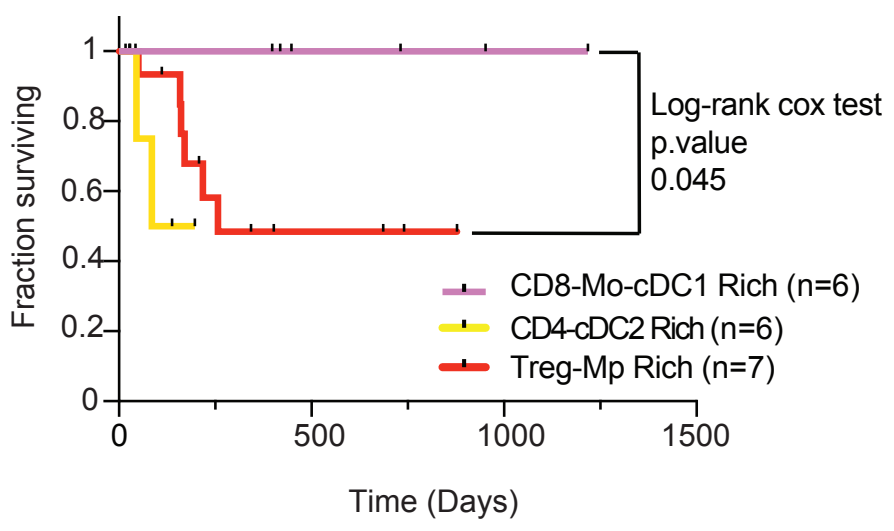


A
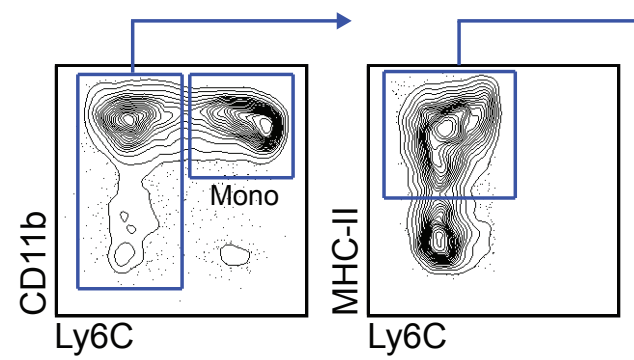

C

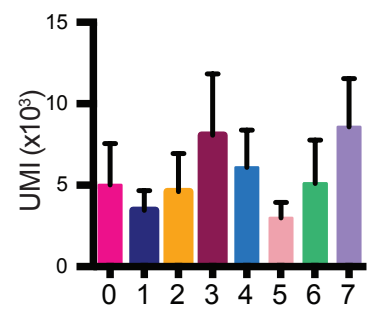

$E$

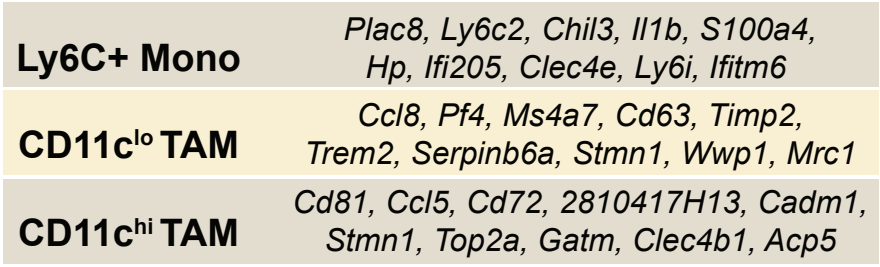

G

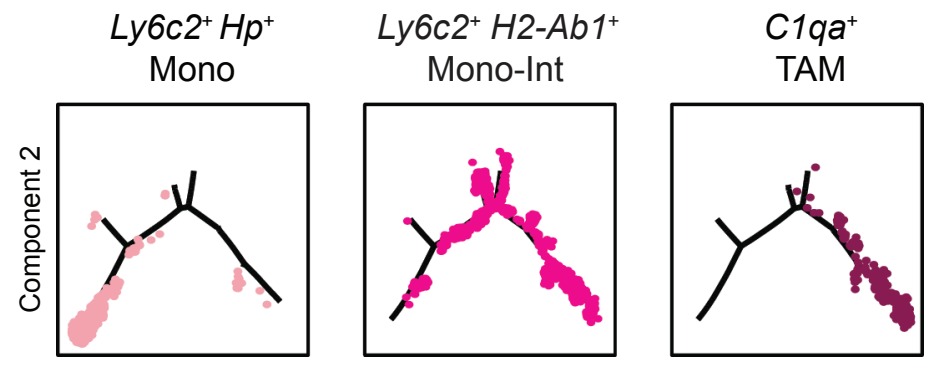

Component 1

IFN-

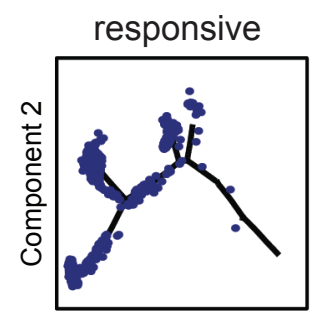

Component 1
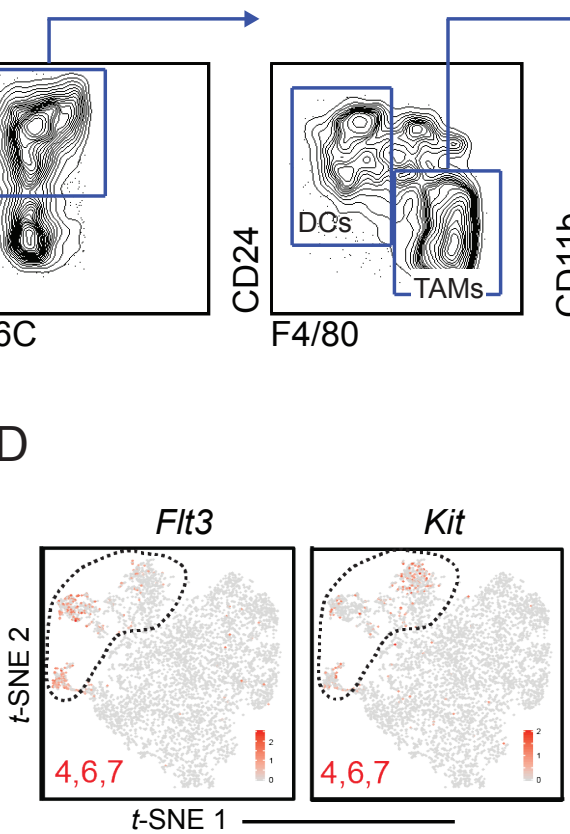

Plac8, Ly6c2, Chil3, II1b, S100a4 Hp, Ifi205, Clec4e, Ly6i, Ifitm6

Ccl8, Pf4, Ms4a7, Cd63, Timp2, d81, Ccl5, Cd72, 2810417H13, Cadm1

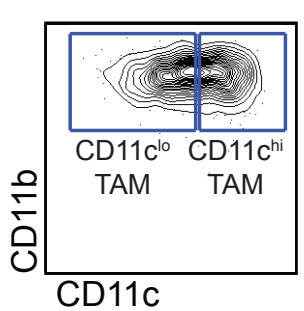

F
B

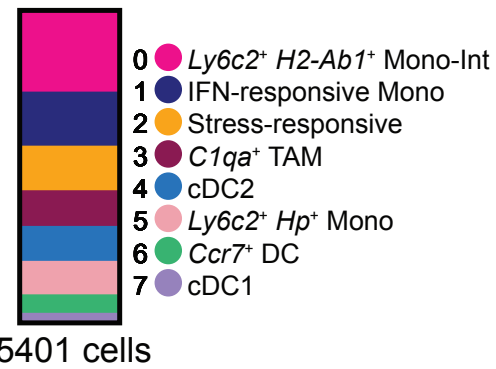

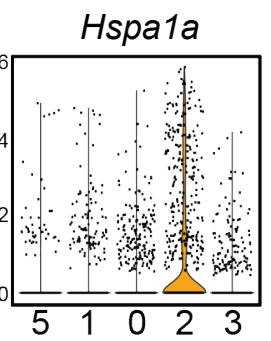

Ero1I
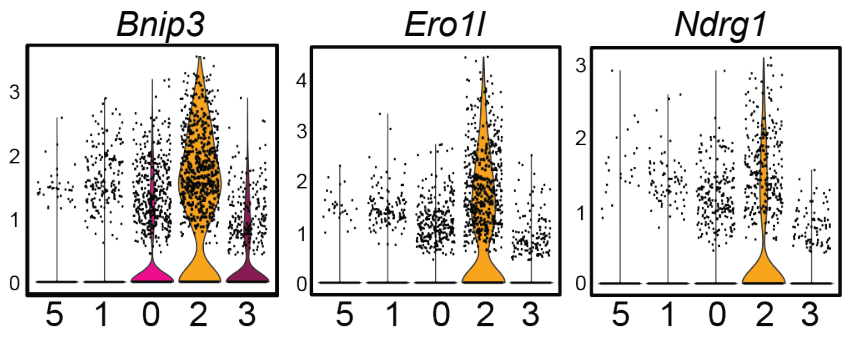

$\mathrm{H}$

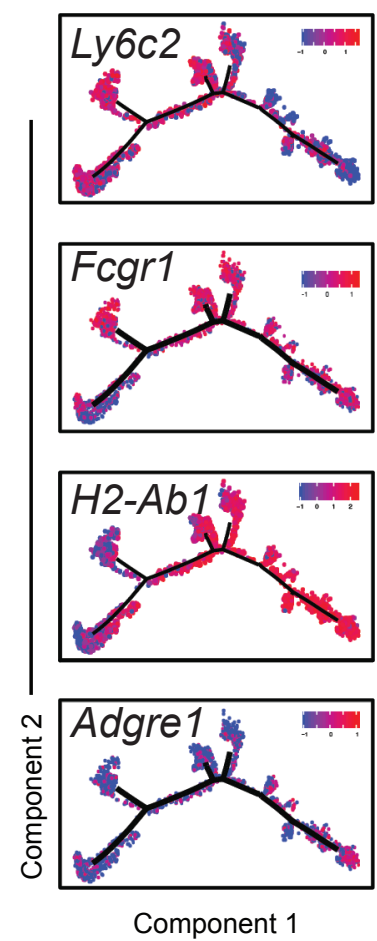

I
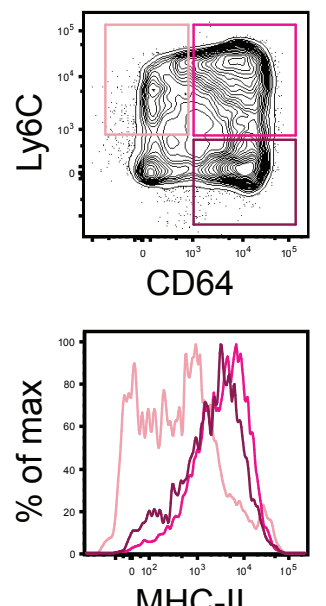

MHC-II

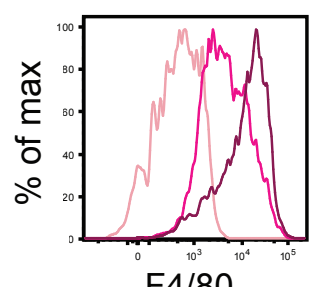

F4/80 
A

Sorted Ly6C ${ }^{\text {hi }}$ Mono

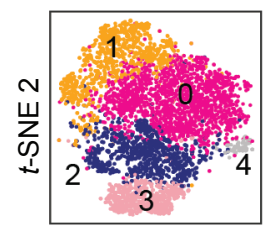

t-SNE 1

C

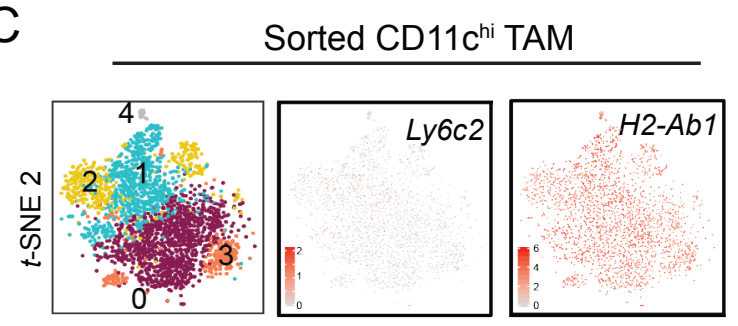

$t$-SNE 1

D

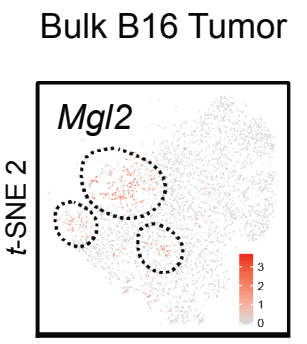

t-SNE 1

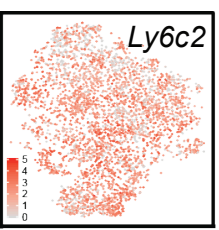

Sorted CD11c $c^{\text {hi }}$ TAM

-

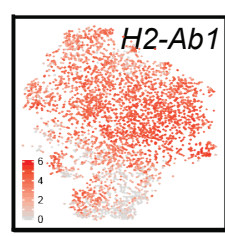

- 
A

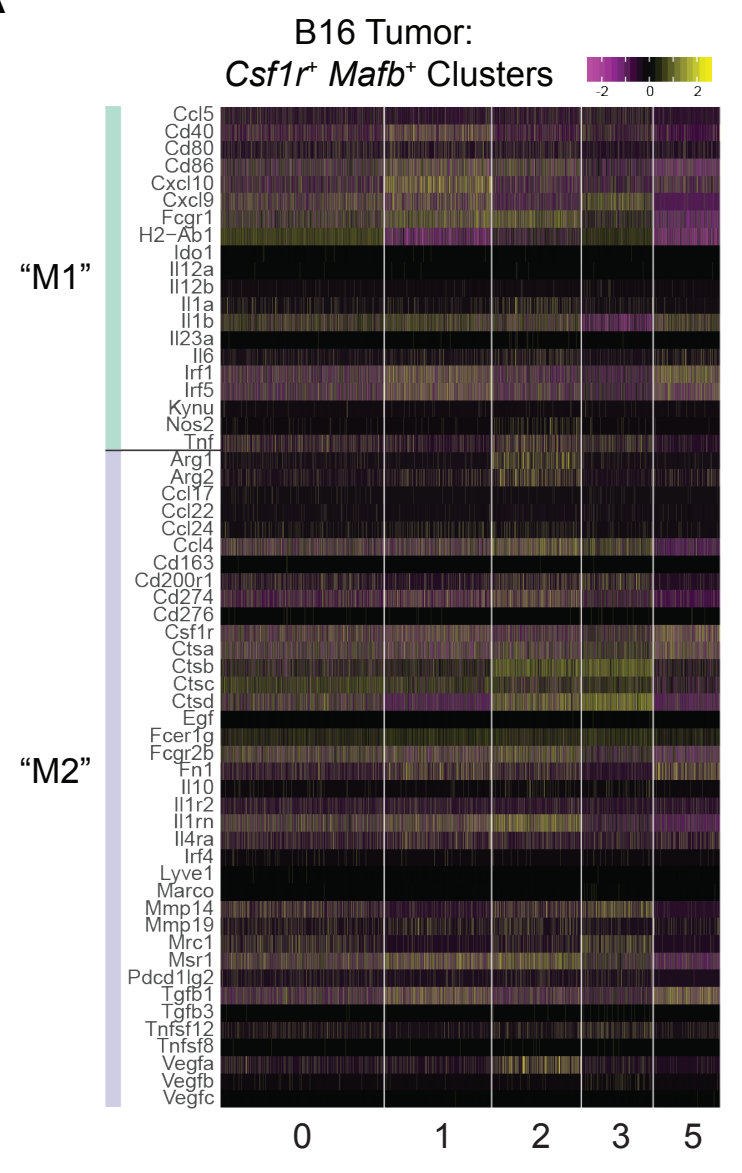

D

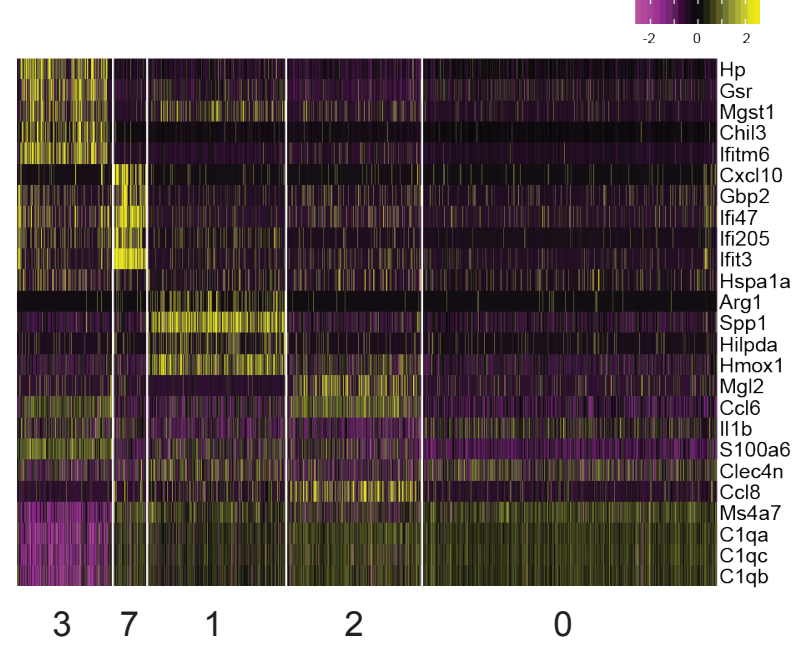

E

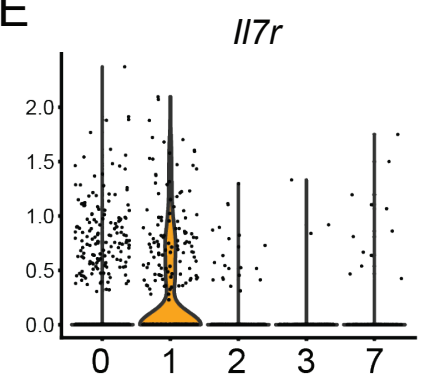

B

C
Vcam1

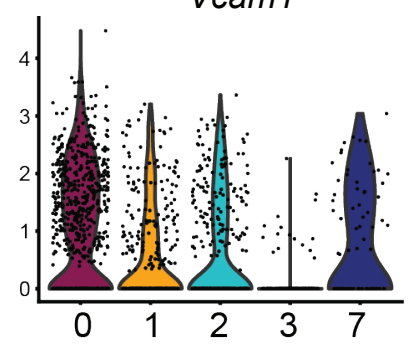

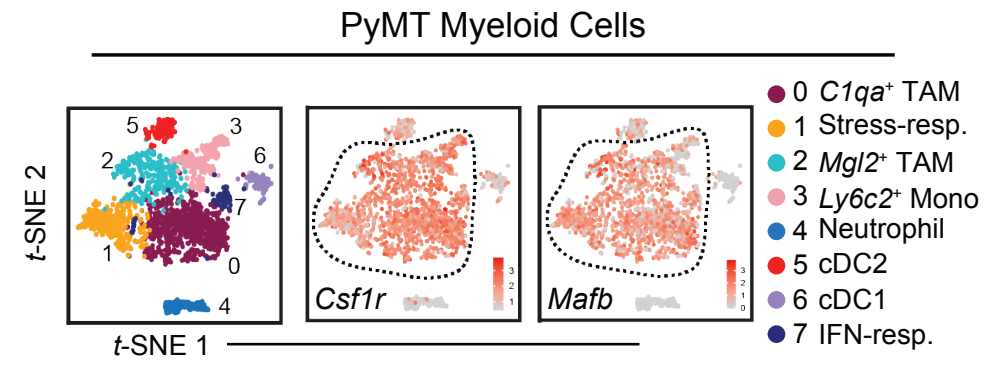

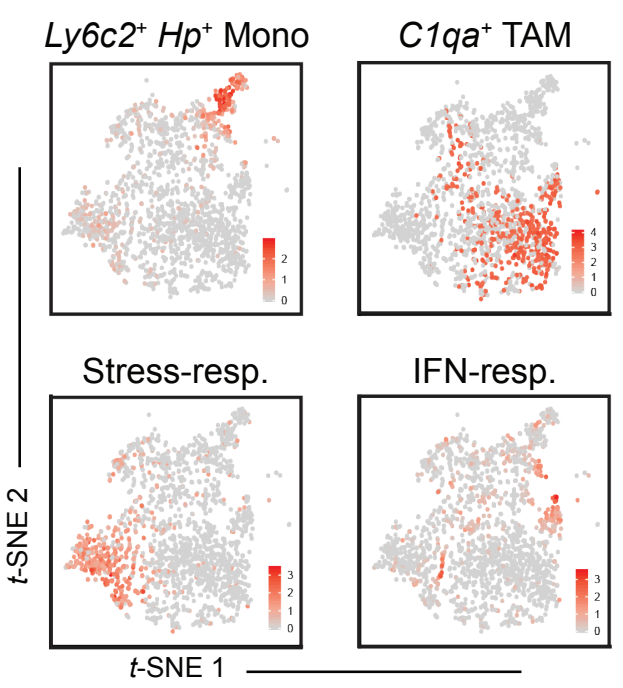

F

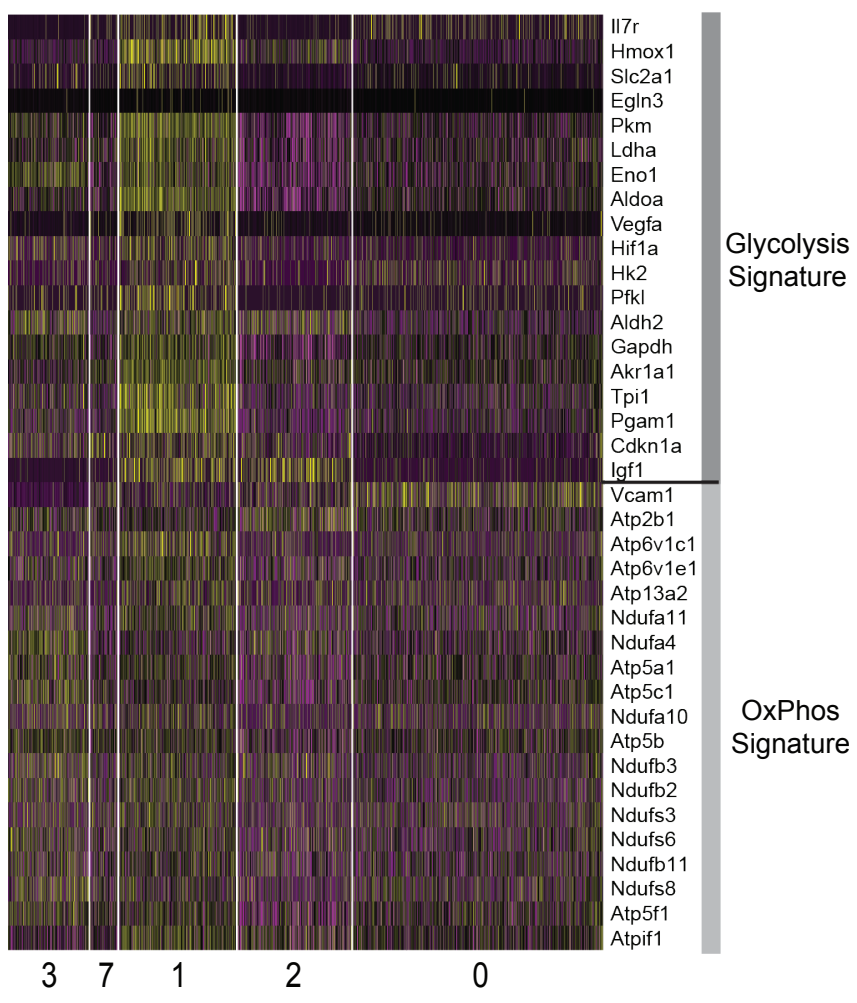


bioRxiv preprint doi: https://doi.org/10.1101/2021.07.07.451502; this version posted July 8, 2021. The copyright holder for this preprint (which was not certified by peer review) is the author/funder. All rights reserved. No reuse allowed without permission.

A
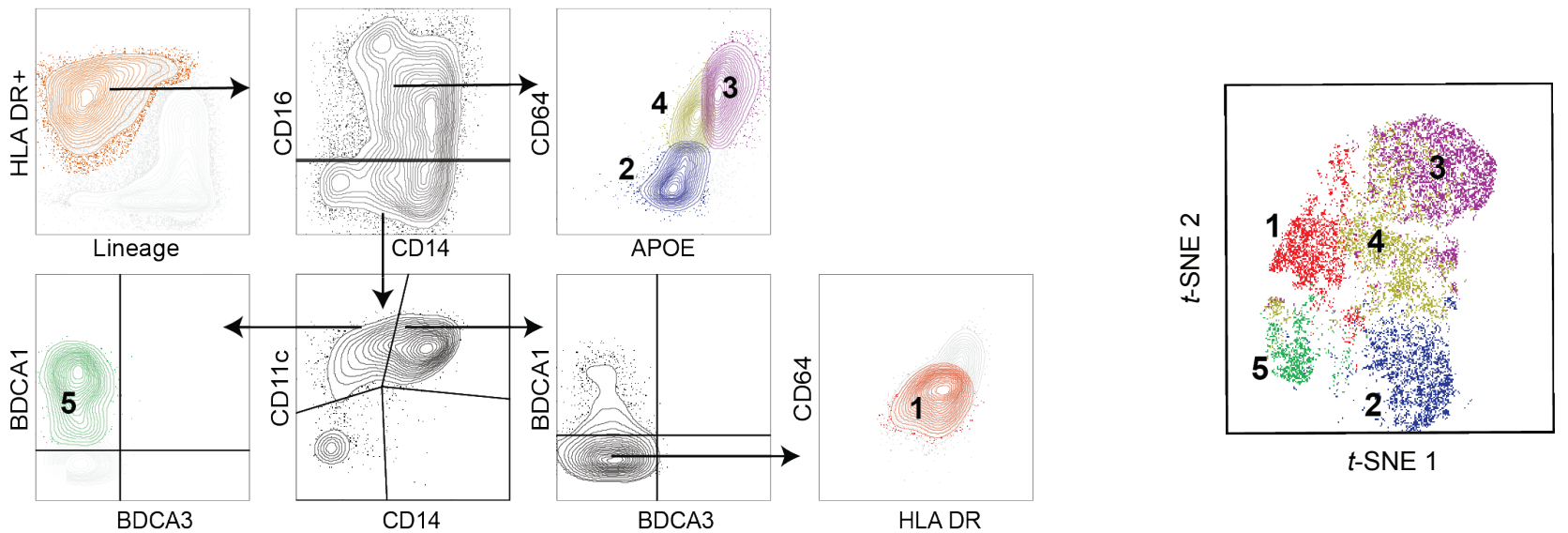

B

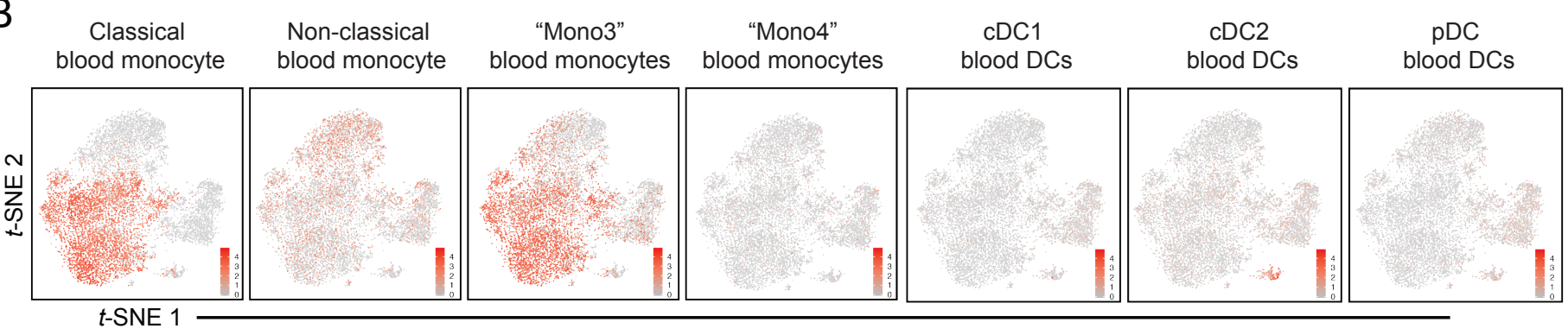

C

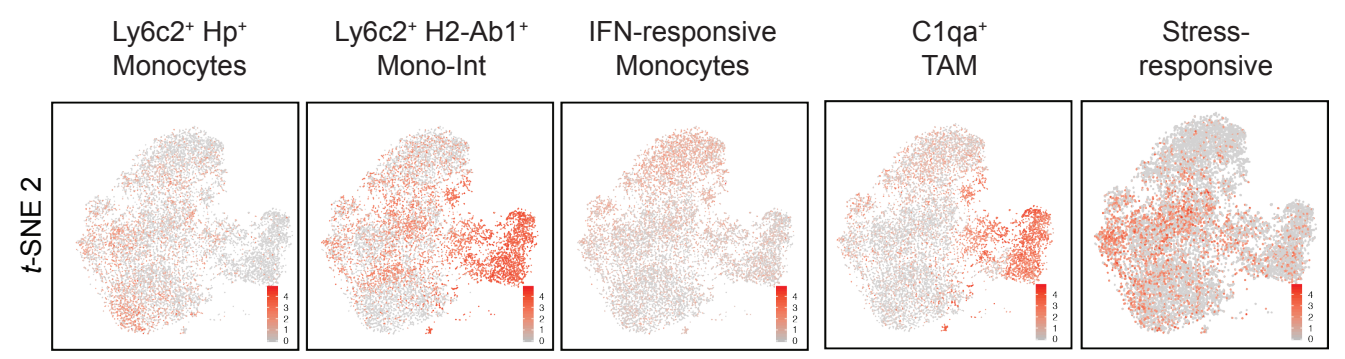

t-SNE 1

D

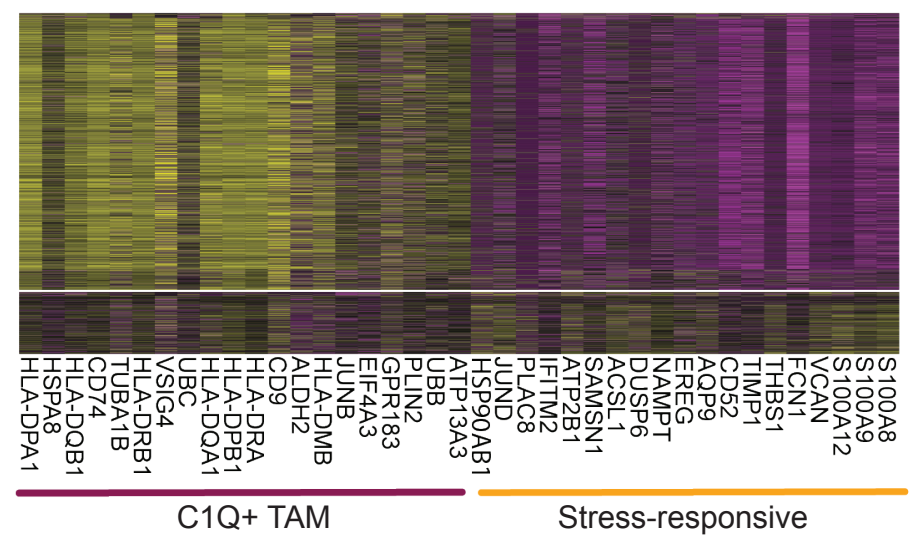

$E$

'M1' Signature

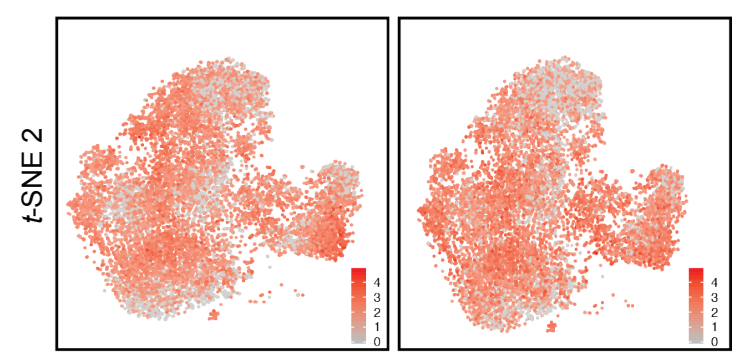

t-SNE 1
'M2' Signature 
A

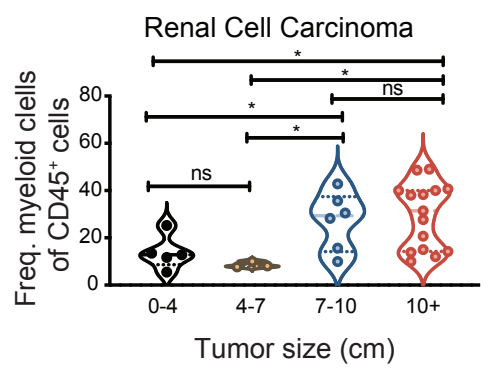

B

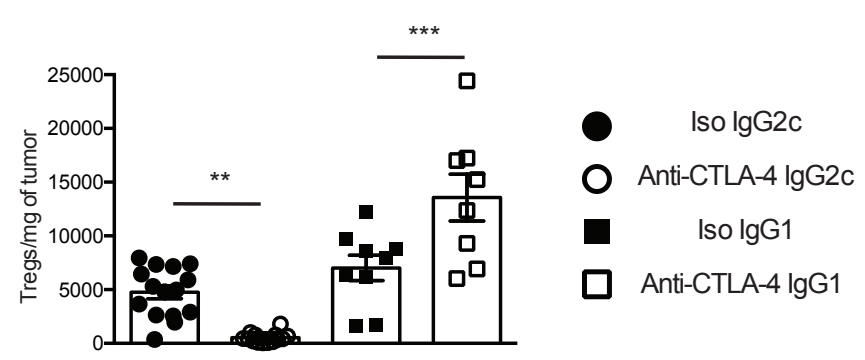

D

Foxp3-DTR

Tumor Myeloid Cells
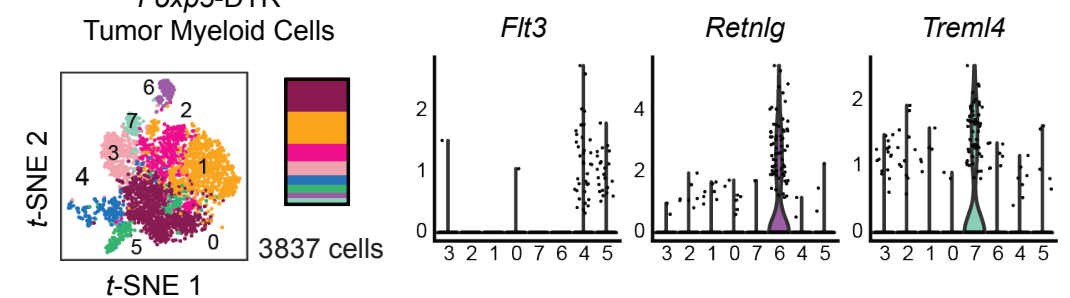

E
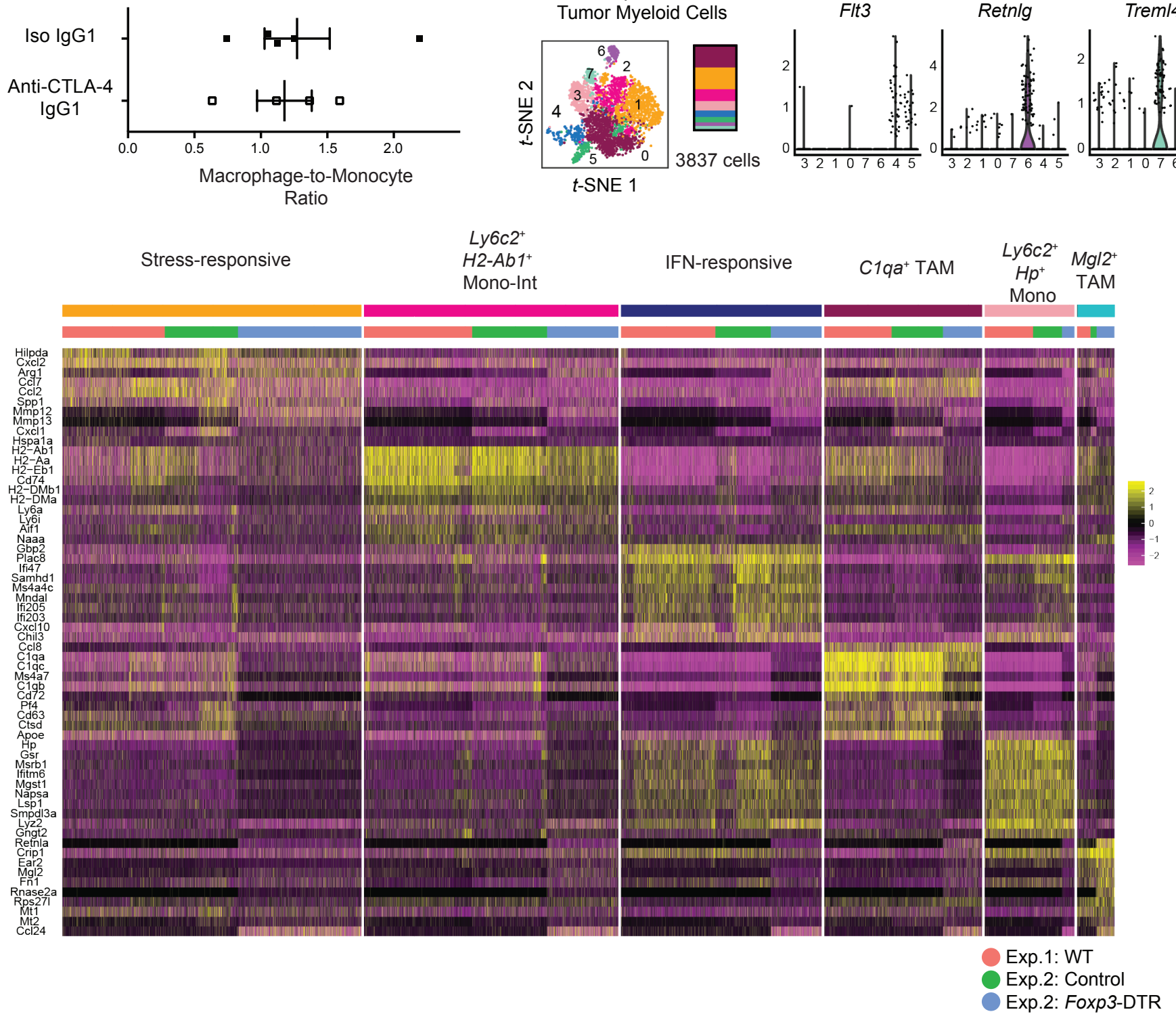

E

F

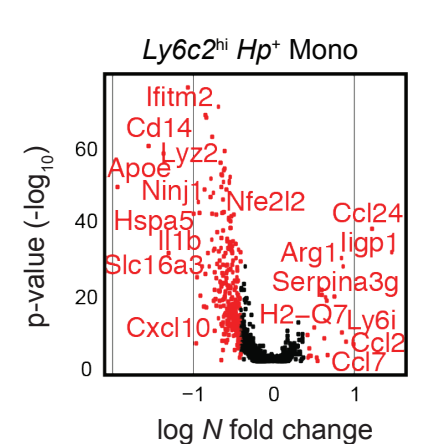

Mujal AM, Combes AJ, et al. 
A

TCGA Overall Survival in Kidney Tumors
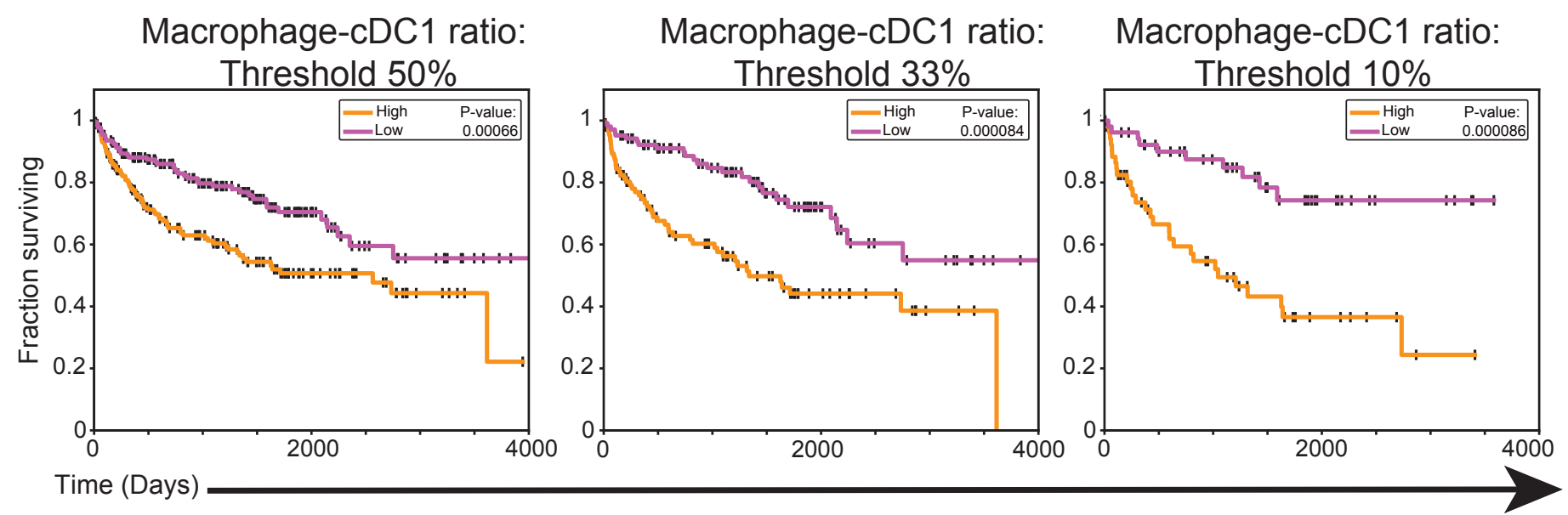

B

$\mathrm{cDC1}$ :

Threshold $10 \%$

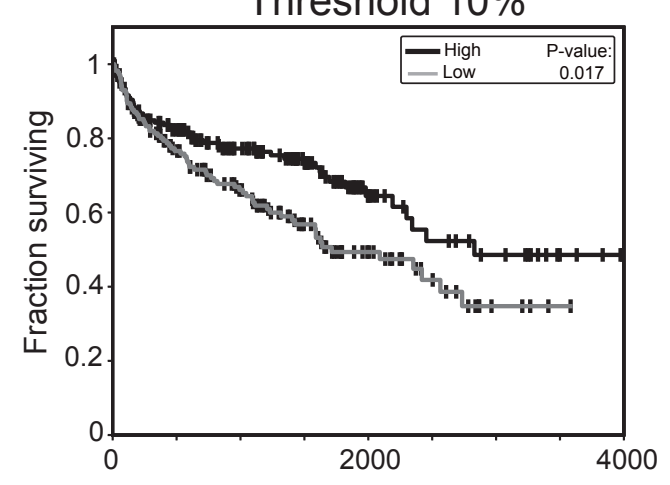

C

\begin{tabular}{|l|c|c|c|}
\hline Signature & Threshold & HR [95\%Cl] & Pvalue \\
\hline Mac/cDC1 & $50 \%$ & 0.77 & $6.66 \mathrm{E}-04$ \\
\hline Mac/cDC1 & $33 \%$ & 0.68 & $8.44 \mathrm{E}-05$ \\
\hline Mac/cDC1 & $10 \%$ & 0.42 & $8.69 \mathrm{E}-05$ \\
\hline cDC1 & $10 \%$ & 0.79 & $1.17 \mathrm{E}-02$ \\
\hline
\end{tabular}

